\title{
Structure-kinetic-relationship reveals the mechanism of selectivity of FAK inhibitors over PYK2
}

Benedict-Tilman Berger ${ }^{1,9,}{ }^{*}$ Marta Amaral ${ }^{2,3, \S,}{ }^{*}$, Daria B. Kokh ${ }^{4 *}$, Ariane Nunes-Alves ${ }^{4,7}$, Djordje Musil ${ }^{2}$, Timo Heinrich ${ }^{2}$, Martin Schröder ${ }^{1,9}$, Rebecca Neil ${ }^{4}$, Jing Wang ${ }^{5}$, Iva Navratilova ${ }^{6}$, Joerg Bomke ${ }^{2}$, Jonathan M. Elkins ${ }^{5}$, Susanne Müller ${ }^{1,9}$, Matthias Frech², Rebecca C. Wade ${ }^{4,7,8}$ and Stefan Knapp ${ }^{1,9,10 \#}$

${ }^{1}$ Structural Genomics Consortium, Goethe University Frankfurt, Buchmann Institute for Molecular Life Sciences, Max-von-Laue-Straße 15, 60438 Frankfurt am Main, Germany

${ }^{2}$ Discovery Technologies, Merck KGaA, Frankfurter Straße 250, 64293 Darmstadt, Germany

${ }^{3}$ Instituto de Biologia Experimental e Tecnológica, Avenida da República, Estação Agronómica Nacional, 2780-157 Oeiras, Portugal

${ }^{4}$ Molecular and Cellular Modeling Group, Heidelberg Institute for Theoretical Studies (HITS), SchloßWolfsbrunnenweg 35, 69118 Heidelberg, Germany

${ }^{5}$ Structural Genomics Consortium, Nuffield Department of Medicine, University of Oxford, Old Road Campus Research Building, Roosevelt Drive, Oxford OX3 7DQ, UK.

${ }^{6}$ Division of Biological Chemistry and Drug Discovery, School of Life Sciences, University of Dundee, Dow Street, Dundee DD1 5EH, UK

${ }^{7}$ Zentrum für Molekulare Biologie der Universität Heidelberg, DKFZ-ZMBH Alliance, Im Neuenheimer Feld 280, 69120 Heidelberg, Germany

${ }^{8}$ Interdisciplinary Center for Scientific Computing (IWR), Heidelberg University, Im Neuenheimer Feld 205, 69120 Heidelberg, Germany

${ }^{9}$ Institute of Pharmaceutical Chemistry, Goethe University Frankfurt, Buchmann Institute for Molecular Life Sciences, Max-von-Laue-Straße 9, 60438 Frankfurt am Main, Germany

${ }^{10}$ German Cancer network DKTK and Frankfurt Cancer Institute (FCl), Goethe University Frankfurt, Frankfurt am Main, Germany

*Authors contributed equally

$\S$ current address: Biologics Research, Sanofi-Aventis Deutschland GmbH, Industriepark Höchst, 65926 Frankfurt am Main, Germany

"Lead contact and Author for correspondence: knapp@pharmchem.uni-frankfurt.de

Keywords: Focal Adhesion Kinase (FAK), Proline-rich Tyrosine Kinase 2 (PYK2), ligand residence time, NanoBRET, kinase inhibitor, $\tau$ RAMD 


\section{Summary}

There is increasing evidence of a significant correlation between prolonged drug-target residence time and increased drug efficacy. Here, we report a structural rational for kinetic selectivity between two closely related kinases: Focal Adhesion Kinase (FAK) and Proline-rich Tyrosine Kinase 2 (PYK2). We found that slow off-rate FAK inhibitors induce helical structure at the DFG motif of FAK but not PYK2. Binding kinetic data, high resolution structures and mutagenesis data support the role of hydrophobic interactions of inhibitors with the DFG helical region providing a structural rational for slow dissociation rates from FAK and kinetic selectivity over PYK2. Our experimental data correlated well with computed relative residence times from molecular simulations providing a feasible strategy for rationally optimizing ligand residence times. We suggest that the interplay between the protein structural mobility and ligand-induced effects is a key regulator of the kinetic selectivity of inhibitors of FAK versus PYK2. 


\section{Introduction}

Focal adhesion kinase (FAK) and proline-rich tyrosine kinase 2 (PYK2) are two closely related non-receptor tyrosine kinases that constitute the subfamily of adhesion kinases (Schlaepfer et al., 1999). Both kinases are involved in the regulation of cell migration, adhesion and survival in response to extracellular signals and they share a similar multidomain organization comprising an $\mathrm{N}$-terminal FERM domain, a central catalytic kinase domain (with 61\% sequence identity), a proline rich (PR) region and a C-terminal focal adhesion targeting (FAT) domain (Lipinski and Loftus, 2010). Despite their homology, signaling events leading to kinase activation differ, with FAK being primarily activated by integrins, growth factor receptors, and cytokine receptors, while PYK2 activation is dependent on intracellular calcium mobilization (Avraham et al., 2000). Focal adhesion kinases promote tumor proliferation and metastasis and that they are overexpressed and upregulated in several types of cancers (Fan and Guan, 2011; Tai et al., 2015; Wendt et al., 2013). PYK2 has been found to be upregulated when FAK activity is suppressed, suggesting compensatory signaling pathways that may result in drug resistance. Efforts have therefore been made to develop dual FAK/PYK2 inhibitors that are currently in clinical testing (Lv et al., 2018).

An interesting series of aminopyrimidines was reported by Pfizer, resulting in the development of dual FAK/PYK2 inhibitors PF-562271 (1) (Roberts et al., 2008), PF-431396 (Buckbinder et al., 2007) and defactinib (PF-8554878 (Jones et al., 2011)), as well as selective FAK (PF-573228 (Slack-Davis et al., 2007)) and PYK2 (PF-719 (Tse et al., 2012)) inhibitors, respectivly. Despite the high sequence similarity of the kinase domains of FAK and PYK2 (Fig. S1), 1 adopts different back pocket binding modes in the two proteins (Fig. 1A). Strikingly, the conserved tripeptide DFG-motif forms an alpha-helical structure in FAK enabling multiple contacts between inhibitor and kinase, notably between the pyridine ring and L567 (Fig. 1B). In PYK2, the same amino acid residues are positioned in a linear conformation with only PYK2-D567 being able to form contacts with the inhibitor (Fig. 1C). This helical conformation of the DFG-motif in FAK has been invoked as providing a possible structural explanation for the approximately 9 -fold greater potency of 1 for FAK $\left(/ C_{50}=1.5 \mathrm{nM}\right)$ over PYK2 $\left(/ C_{50}=13 \mathrm{nM}\right)$ (Roberts et al., 2008).

Recently, several studies have suggested that the kinetic properties of a drug, specifically the residence time ( $\tau=1 / k_{\text {off }}$ ), may provide a better indication of the duration of the pharmacodynamic action in vivo than binding affinity alone (Copeland, 2016; Schuetz et al., 2017). The observation of a helical conformation in FAK but not PYK2 suggests that it may provide a mechanism for the kinetic selectivity of 1. The structurally very similar dual FAK/PYK2 inhibitor PF-431396 (10 - with phenylsulfonamide instead of the aminopyridine in 1$)$ shows a similar selectivity in potency $\left(\right.$ FAK $I C_{50}=1.5 \mathrm{nM}$ over PYK2 $\left.I C_{50}=11 \mathrm{nM}\right)$ 
(Roberts et al., 2008). As 10 was also reported to show fast binding kinetics on PYK2 $\left(k_{\text {on }}>10^{6} \mathrm{M}^{-1} \mathrm{~s}^{-1}, k_{\text {off }}>\right.$ $10^{-2} \mathrm{~s}^{-1}$ ) (Han et al., 2009), we hypothesize that the about 7-fold selectivity for FAK - and therefore as well the about 9-fold selectivity (Roberts et al., 2008) of 1 - may be related to very slow binding kinetics to FAK. As selectivity is often hard to achieve for related protein kinases, an understanding of the mechanisms of kinetic selectivity of inhibitors of FAK and PYK2 would be beneficial for future medicinal chemistry efforts.

Therefore, in this study, we explored the structural features that modulate the kinetic properties of a series of FAK and PYK2 inhibitors to support the development of kinetically selective or balanced dual FAK/PYK inhibitors. In order to gain a mechanistic understanding of the distinct kinetic profiles of FAK and PYK2 binding, we performed surface plasmon resonance assays, binding experiments in cells, structural and sitedirected mutagenesis studies, conventional molecular dynamics (MD) and $\tau$ random acceleration molecular dynamics ( $\tau$ RAMD) simulations. This combination of techniques allowed us to relate the structural and dynamic differences between the two kinases to the kinetic selectivity of the inhibitors.

\section{Results and Discussion}

\section{Design of a series of inhibitors to probe the structure activity relationship in FAK and PYK2} Based on the comparison of the crystal structures of $\mathbf{1}$ in complex with FAK and PYK2 (Fig. 1), we designed and synthesized a series of inhibitors to probe the influence of interactions with different parts of the ATP binding site on binding kinetics in FAK and PYK2. The central amino-pyrimidine hinge binding scaffold was modified at three positions, probing interactions with the solvent exposed pocket and interactions with the non-conserved upper lobe residues $\left(\mathbf{R}^{\mathbf{1}}\right)$, the DFG helix induced in FAK after ligand binding $\left(\mathbf{R}^{\mathbf{2}}\right)$, as well as the back pocket interface with the induced helix in FAK $\left(\mathbf{R}^{3}\right)$ (

Table 1). For a first assessment of the effect of the modifications on inhibitor binding kinetics and affinity, we performed surface plasmon resonance (SPR) measurements.

In order to develop inhibitors that show different binding kinetics between these two related kinases, we were particularly interested in the role of the hydrophobic interaction of the inhibitor with the induced helix located C-terminal to the FAK DFG motif (residues D564-G566) which positioned FAK-L567 into the ATP binding site. The pyridyl-methansulfonamide in $\mathbf{R}^{1}$ was therefore replaced by diverse aromatic moieties (compounds 5-12) modulating the electron density of the pendant aromatic ring system, as well as substitution patterns in the ring.

Another interaction unique to FAK was a polar contact of the oxindole of 1 with FAK-R426. Despite conservation of this residue in PYK2 (R429), this interaction was not observed in crystal structures of this 
kinase. To probe the contribution of this structural difference on binding kinetics, we introduced single aromatic ring systems instead of the oxindole moiety in 1, including a phenyl moiety (3), a ring expanded 3,4 dihydroquinoline-2-one (4 and 5), N-methylbenzamide (11, defactinib) and Nmethylbenzenesulfonamide (12). While we did not obtain data for a substitution of the oxindole in $\mathbf{R}^{1}$ with dihydroquinoline-2-one in $\mathbf{4}$ we observed a comparable potency to $\mathbf{1}$ by introducing a methylsulfonylbenzene in $\mathbf{R}^{1}(\mathbf{5})$. In position $\mathbf{R}^{3}$, only a $-\mathrm{CF}_{3}$ group and a $\mathrm{CF}_{3}$-deleted variant $(-\mathrm{H}, \mathbf{2})$ were tested. The removal of the trifluoromethyl group in $\mathbf{2}$ resulted in a significant reduction of affinity compared to PF-562271 (1), associated with shorter residence time as expected for the key interaction of the trifluoromethyl group with the back pocket. The trifluoromethyl group was therefore kept in all other inhibitors that we investigated.

\section{L567-ligand interactions increased potency and prolonged residence times in FAK}

Modifications in the selected inhibitor set, significantly modulated binding affinity for FAK and PYK2. Interestingly, introducing a phenylsulfonamide moiety at position $\mathbf{R}^{\mathbf{1}}$ in $\mathbf{1 0}$ resulted in a 4 -fold improvement in potency for FAK compared to the parent compound $\mathbf{1}$. We hypothesized that the benzene ring of 10 provided a more favorable hydrophobic interaction with L567 compared to the pyridine ring in

1. Indeed, if the polarity of the ring system was increased by incorporation of two nitrogen atoms as exemplified in 11, the affinity decreased further. The corresponding change in off-rate was a result of a difference in binding free energy, as the on-rates of 1, 10 and 11 were similar. For PYK2, we observed similar affinities and kinetic parameters for $\mathbf{1}, \mathbf{1 0}$, and $\mathbf{1 1}$, in agreement with the lack of the induced helical structure and interaction with the conserved PYK2-L570.

The introduction of a sulfonylphenyl moiety at $\mathbf{R}^{\mathbf{1}}(\mathbf{5})$ resulted in a decreased affinity for FAK. This modification was accompanied by a 2 -fold increase in the inhibitor off-rate. Compared to 1 , the removal of the sulfonamide in $\mathbf{8}$ led to a decrease in binding affinity and interestingly, a substitution of the pyridine ring in 8 with benzene (9) or para-fluorobenzene (7) was not tolerated and resulted in an affinity loss of 350-fold and 1300-fold, respectively. Introduction of an indoline-2-one ring system was however tolerated (6) maintaining low nanomolar affinity. Similar effects on inhibitor binding affinity by $\mathbf{R}^{\mathbf{1}}$ substitutions were observed for PYK2.

Interestingly, the $\mathbf{R}^{\mathbf{2}}$ substituent played a key role in determining both potency and the binding kinetics profile. Changing the oxindole ring in $\mathbf{1}$ to a phenyl (3) resulted in a 93-fold decrease in potency and a 60fold drop in the on-rate, most likely because the phenyl ring does not allow the formation of a hydrogen 
bond with FAK-R426. Inhibitor 12 has been reported as a PYK2 selective inhibitor (Tse et al., 2012) and we indeed measured tighter binding to PYK2 but observed only about 6-fold selectivity against FAK.

Comparing the selectivity profiles of both FAK and PYK2, it was intriguing that the $k_{\text {on }}-k_{\text {off }}$-plot (Fig. S3) clearly demonstrated that most inhibitors had very similar association rate constants $k_{\text {on }}$. Remarkably, the variation was less than 10 -fold over all inhibitors tested. As a result, the different binding affinities for these two kinases were mainly due to differences in the dissociation rate constant $k_{\text {off. }}$ Interestingly, despite similar $I C_{50}$ values, we observed that 10 showed a dissociation rate from FAK of 138 min - more than two orders of magnitude slower than that reported for PYK2 (Han et al., 2009).

\section{Slow off-rate inhibitors induce a helical conformation of the activation loop in FAK}

In order to better understand the structural mechanisms giving rise to the different binding kinetics of this inhibitor series, we solved six crystal structures of FAK in complex with different aminopyrimidine derivatives, including the most interesting substitutions in $\mathbf{R}^{\mathbf{1}}, \mathbf{R}^{\mathbf{2}}$ and $\mathbf{R}^{\mathbf{3}}$ (Fig. 2).

The structure of the inhibitor with the slowest dissociation off-rate - $\mathbf{1 0}$ - in complex with FAK confirmed the overall binding mode previously described for 1 (Roberts et al., 2008). 10 formed hydrogen-bonds between the inhibitor and the backbone of C502 located in the hinge region. The oxygen atom of the amino-oxindole $\left(\mathbf{R}^{2}\right)$ was within hydrogen-bonding distance of the side chain of R426. The trifluoromethyl group $\left(\mathbf{R}^{3}\right)$ bound to the cavity created by the sidechains of M499, D564 and L567. The phenyl-sulfonamide $\left(\mathbf{R}^{1}\right)$ formed a hydrophobic interaction with L567. Finally, the sulfonamide was stabilized by a hydrogen bond with the backbone amide of FAK-D564. The conformation of this helix and the binding mode of the inhibitor series discussed here were similar to that reported in the structure of the complex with the clinical FAK inhibitor TAE226 (Fig. S2) (Lietha and Eck, 2008).

In addition, we also determined structures in complex with $\mathbf{3}$ and $\mathbf{5}$, harboring a sulfonamide moiety in $\mathbf{R}^{\mathbf{1}}$. Intriguingly, all structures with these slow off-rate inhibitors revealed an induced helical structure in the activation loop region. The structure in complex with $\mathbf{4}$ induced a helical conformation in FAK. Therefore, we assumed similar kinetics compared to $\mathbf{1}$. Interestingly, for 5, the FAK-L567 interaction was mainly due to a $180^{\circ}$ rotated T-shaped $\pi$-stacking interaction of the aromatic ring system based on the shorter distance provided by the phenylsulfodioxide without the additional atoms present in $\mathbf{1}$ and additionally formed a hydrophobic interaction with L553.

We observed that the absence of the $\mathbf{R}^{1}$ methyl sulfonamide group in $\mathbf{8}$ prevented the formation of the DFG-motif helix and this inhibitor was shown to have an increased off-rate compared to 1 . The oxindole 
ring of $\mathbf{1}$ and $\mathbf{1 0}$ in position $\mathbf{R}^{\mathbf{2}}$ interacted with FAK R426 via a hydrogen bond. Changes in the $\mathbf{R}^{\mathbf{2}}$ position did not lead to different interactions of the $\mathbf{R}^{\mathbf{2}}$ groups. However, while the oxindole moiety was within hydrogen bonding distance of FAK R426, this interaction appeared transient and was not present in all chains in the asymmetric unit. This makes an involvement in the modulation of the inhibitors' on-rate less likely. We were successful in solving the structure of an inhibitor harbouring different moieties in $\mathbf{R}^{\mathbf{3}}$, which pointed towards the back pocket. Analysis of $\mathbf{2}$ in complex with FAK revealed significant differences in the pyridyl-methansulfonamide group orientation when compared to $\mathbf{1 0}$ and $\mathbf{1}$, which in the case of $\mathbf{2}$ did not allow a direct interaction with the DFG-motif. Hence, its conformation was the canonical DFG-in arrangement. The trifluoromethyl group was involved in hydrophobic interactions with M499, D564, and L567 in the structures of inhibitors 1 and 3-11. The lack of these hydrophobic interactions in 2 explained the impact of this substituent on binding affinity and fast binding dissociation kinetics.

As expected from the SAR and SKR analysis, the slowest off-rate inhibitors of FAK, $\mathbf{5}$ and 10, formed similar interactions as reported for $\mathbf{1}$. In particular, the DFG motif was stabilized in a helical conformation by hydrophobic interactions with FAK L567. Simulations suggested that the very potent and slow off-rate compound $\mathbf{1 1}$ had a similar binding mode to that one observed experimentally for $\mathbf{1 0}$ and $\mathbf{1}$. All the inhibitors contained a sulfoxide moiety coupled to an aromatic ring system in $\mathbf{R}^{\mathbf{1}}$. The overall results revealed some general trends in inhibitor binding characteristics as a function of the structural rearrangement of the DFG motif. Compounds that induce a helical conformation of the DFG motif via a hydrophobic interaction with FAK-L567 had significantly slower binding kinetics and higher potency when compared to compounds that assumed a type-I binding mode.

\section{The kinetic selectivity profile of FAK and PYK2 is dictated by the formation of a helical conformation of the DFG motif}

We compared our structural data of FAK with published structures of PYK2 in complex with 1 (PDB 5tob) (Fig. 1), 10 (PDB 3fzr, Fig. 3A) and 12 (PDB 3h3c, Fig. 3B) to identify crucial interaction residues that are relevant for the kinetic selectivity for either FAK or PYK2.

The superimposed crystal structures of FAK and PYK2 in complex with $\mathbf{1 0}$ showed a similar conformation of the amino-methyl-phenyl-sulfonamide group as that reported for $\mathbf{1}$. In contrast to an interaction of $\mathbf{1 0}$ with the helical DFG motif in FAK, this interaction is missing for PYK2. The interaction of 10 with PYK2 is characterized by a DFG-out conformation and hydrophobic interactions with residues preceding the Ploop (L431, G432) similar to FAK. We hypothesized that the differences between the conformations of the DFG motif are likely to contribute to differences in potency and paralogue selectivity of the trifluoromethyl 
pyrimidine series, as previously reported (Heinrich et al., 2013; Roberts et al., 2008). The superimposed crystal structures of FAK and PYK2 also revealed a different conformation of the P-loop and a different position of $\alpha \mathrm{C}$. Although this region did not directly interact with the inhibitor, the difference in one particular amino acid residue of the P-loop region, F435 (PYK2) vs. Q432 (FAK) (Fig. S1), might impact the stabilization of the helical conformation of the DFG-motif in FAK, as Q432 interacted with the backbone of G566. While FAK-R426 showed an interaction with 10, the corresponding arginine R429 in PYK2 was not within hydrogen-bonding distance.

In contrast to 10, which showed a 9-fold selectivity for FAK over PYK2, 12 shared the amino pyrimidine scaffold, but harbored different moieties $\mathbf{R}^{\mathbf{1}}$ and $\mathbf{R}^{\mathbf{2}}$ and has been reported as a PYK2-selective inhibitor (Tse et al., 2012). We solved the crystal structure of 12 with FAK to provide structural insights into the mechanism of this selectivity. Exchanging $\mathbf{R}^{\mathbf{1}}$ and $\mathbf{R}^{\mathbf{2}}$ residues in contrast to $\mathbf{1 0}$, resulted in a shift in the position of the sulfonamide, which interacted with R426 (FAK) in $\beta 1$ in both kinases. An interaction of the inhibitor with the conserved leucine residue L567 or the respective L570 in PYK2 was missing in both FAK and PYK2 stabilizing a DFG-in conformation. The FAK DFG motif did not form the helical secondary structure in complex with 12 - as seen for 10 - supporting the idea that differences in the arrangement of the DFG motif were relevant for the inhibitor selectivity of $\mathbf{1 2}$ for PYK2.

\section{Protein flexibility and the dynamics of the protein-ligand interactions differ for FAK and PYK2}

To investigate the origin of the differences in the binding rates between FAK and PYK2 for the set of compounds considered, we performed MD simulations. We carried out both conventional MD simulations of the protein-ligand complexes and $\tau$ RAMD (Kokh et al., 2018) simulations of ligand dissociation for all 12 compounds binding FAK and for the 10 compounds whose binding to PYK2 was observed experimentally. Conventional MD simulations of the protein-ligand complexes showed that the ligands and the parts of the protein that line the binding site (in particular the $\beta 1, \alpha C$, and DFG-motif regions) generally showed more mobility in PYK2 than in FAK (Fig. 4A). The DFG-motif region was highly mobile for the complexes with compounds 1 and $\mathbf{3}$ in PYK2 simulated starting from the crystal structure with PDB 5tob, where the position of the phenylalanine of the DFG fragment is different from in other PYK2 crystal structures and the side chain position was found to be more variable in MD simulations (for details, see Fig. S4).

To explore the possible reasons for the structural stability of FAK with respect to PYK2, we compared protein-ligand interaction fingerprints in the MD trajectories. Although the pattern of protein-ligand interactions in trajectories for PYK2 and FAK was generally similar (Fig. 4B, C), there were several differences. First, with exception of compound 2, an additional hydrogen bond with D564 appeared in the 
FAK complexes with compounds that have a methane sulfonamide $\mathbf{R}^{1}$ moiety oriented towards the DFGmotif (see Fig. 4D).

The number and stability of protein-ligand hydrophobic interactions is larger in FAK. These interactions included not only the DFG-motif (D564/D567,L569/570), having a helical conformation in FAK but also interactions with residues V484 located deep in the pocket and L553 in addition to interactions with conserved residues V436/V439 and A452/A455 (Fig. 4D). Additionally, the hinge residue L501 in FAK formed a temporary contact with most ligands. This suggests a tighter packing of the ligand in the binding pocket of FAK than PYK2. Indeed, most of the compounds bound to PYK2 form a water-mediated hydrogen bond with D567 through the $\mathbf{R}^{1}$ moiety, which indicates that the pocket is relatively open and partially hydrophilic. For FAK, this interaction is observed only for $\mathbf{2}$ and $\mathbf{6}$ which bound to the helical DFG-loop conformation. In agreement with our structural analysis, we did not observe stable hydrogen bonding to FAK R426 from the oxygen in the oxindole ring and R426 was only transiently involved in a direct or watermediated hydrogen bond with ligands (Fig. 4B).

Next, we employed the $\tau$ RAMD procedure to simulate ligand unbinding and to evaluate the relative residence times of the compounds studied. The computed $\tau$ RAMD residence times overall correlate well with SPR data ( $R^{2}=0.91$ for the whole set of compounds, Fig. $4 E$ ), including those modelled by similarity or docked (such as 6, $\mathbf{7}$ and $\mathbf{1 1}$ for FAK, and most compounds for PYK2, see Methods). The compounds that dissociate more slowly from FAK were well separated from those with faster dissociation rates. However, the ranking of the fast dissociating compounds $\left(k_{\text {off }}>0.03 \mathrm{~s}^{-1}\right)$ was less accurate which was potentially due to the uncertainty in the structure of the modelled complex, as only a few crystal structures of PYK2 (1, 10 and 12) were available (see, for example, the results of simulations with different docking positions for compounds 3 and 9 bound to PYK2 and FAK, respectively, Fig. S4).

All compounds with a sulfonamide moiety at $\mathbf{R}^{1}$ bound to the FAK complex with a helical DFG-motif conformation, formed hydrogen bonds with the DFG-motif backbone (residue D564, Fig. 4D), and showed long residence times $\left(\mathrm{k}_{\text {off }}<0.01 \mathrm{~s}^{-1}\right)$. This interaction, along with the hydrophobic contact with L567, seemed to be critical for residence time prolongation. Indeed, for two fast dissociating compounds, $\mathbf{7}$ and $9\left(k_{\text {off }}>0.05 \mathrm{~s}^{-1}\right)$, with aromatic $\mathbf{R}^{1}$ substitutions, we did not observe differences in residence times when the simulated ligands were docked into the protein structure with a helical or extended conformation of the DFG-motif (see for example, simulation results for compound $\mathbf{9}$ for different conformations of the protein binding site in Fig. S4). Importantly, both compounds formed a hydrophobic contact with L567 in the helical DFG-loop structure, although it was much less stable than in the case of compounds with a sulfonamide moiety at $\mathbf{R}^{\mathbf{1}}$. One more interesting feature was observed in the series of simulations for 
compound 9: the ligand docking positions for which a transient contact with R426 was possible were found to dissociate several fold slower than those for which this hydrogen bond was not observed (see Fig. S4). Thus, both protein structural differences (tighter pocket and more hydrophobic contacts in FAK) and the ligand's ability to form additional hydrogen bonds appeared important for slow dissociation rates.

\section{Binding potency and kinetics of FAK and PYK2 in living cells}

In order to validate the in vitro selectivity and slow binding kinetics of the present series of compounds for FAK versus PYK2, we assessed target engagement and binding kinetics in living cells under near physiological conditions using the NanoBRET technology (Robers et al., 2015). For the target engagement assays, the apparent tracer affinity values of tracer K5 (Vasta et al., 2018) were determined (46 nM and $78 \mathrm{nM}$ for FAK and PYK2, respectively) and used as input parameters in the assay setup for FAK and PYK2 (Fig. S5). Representative results of the target engagement assay for compounds $\mathbf{1 0}$ and $\mathbf{1}$ are shown in Fig. 5A. The intracellular apparent affinities $(6,7)$ were generally 10 -times weaker than the binding affinities calculated from the in vitro SPR measurements (Fig. 5B). NanoBRET data were in very good agreement with previously reported $I C_{50}$ values (Allen et al., 2009; Heinrich et al., 2013; Walker et al., 2008; Walker et al., 2009) for both FAK and PYK2. FAK in vitro selectivity for the present series of compounds was translated into the cellular context, where $\mathbf{5}$ was the most selective compound for FAK with a 330-fold difference compared to PYK2.

Next, we investigated the kinetic aspects in the cellular context using the NanoBRET technology in a washout format. The tracer used had the required rapid binding kinetics, making it suitable for the kinetic analysis of slower-binding inhibitors (Fig. S5). After incubation of the individual inhibitors with their target at a concentration of 10 -fold of their respective $I C_{50}$ to achieve $90 \%$ target occupancy, we removed the unbound compound by wash-out and added the tracer whose association was monitored over time (Fig. S5, Table S3). Our residence time analysis by NanoBRET revealed remarkably slow dissociation rates for 10 on FAK compared to PYK2 and distinguishably slower kinetics for 1 on FAK. For PYK2, in agreement with our SPR data, no inhibitors with slow off-rates were identified in cells. We confirmed that compounds 1 and 10, which had the longest residence times among the entire series of pyrimidines in vitro, also had slow binding kinetics (Fig. 5C) in cells. As expected from the SPR data, compound $\mathbf{1 0}$ showed fast binding kinetics for PYK2, providing an indication for kinetic selectivity. The observed rank-order of intracellular residence times was in good agreement with the residence times determined in SPR experiments. It is noteworthy that in the NanoBRET assay, compound $\mathbf{4}$ showed a very similar potency to compound $\mathbf{1}$ and 
an even longer half-life of dissociation. Together with the relative residence time computations (Fig. 4E), these data indicate a similar kinetic behavior of 4 compared to 1 .

Interestingly, although $\mathbf{5}$ had a higher selectivity for FAK over PYK2, 10 showed longer occupancy due to its slow dissociation kinetics, providing evidence that the residence time can be a valuable indicator of target occupancy.

\section{Structural mechanisms affecting the protein binding site flexibility in FAK and PYK2}

Analysis of the crystal structures and the protein-ligand interaction contacts in MD simulations suggests that the protein structure with a helical DFG-motif conformation formed a tighter binding pocket and enabled a larger number of hydrophobic contacts between the protein and the ligand relative to that with a loop DFG-motif conformation. This analysis did not explain why PYK2 was unable to adopt the helical DFG-motif conformation to form complexes with higher affinity. A possible explanation for such structural differences are sequence variations between FAK and PYK2 (Fig. S1), which may cause differences in protein flexibility. The DFG-motif and several of the following residues are conserved in PYK2 and FAK, whereas in other regions of the binding site, in particular in $\beta 1$ and the hinge region, some sequence variations might affect domain plasticity.

The most notable specific interaction observed in the crystal structures of FAK was a hydrogen bond between the sidechain amide nitrogen of Q432, located in the P-loop, and the backbone oxygen atom of L567, located next to the helical DFG motif (Fig. 6A). This interaction is not possible in PYK2 because the hydrophobic F435 is at the Q432 position (Fig. 6B), although a hydrophobic residue at this position is unusual among the non-receptor kinases (Fig. S6). To explore whether a residue corresponding to Q432 in FAK could stabilize the PYK2 protein structure, we compared MD simulations of the apo wild-type PYK2 with F435Q and F435A mutants. Although the PYK2 mutation F435Q was slightly more stable (Fig. S6), neither coiling of the DFG-motif loop nor contact between Q435 and the DFG-motif region were observed in the MD trajectories, most likely because this would require a much longer simulation time. We also did not observe any stable aromatic stacking of F435 with the surrounding aromatic residues in PYK2, which might affect the position of the P-loop in PYK2, although the $Q$ and A residues at position 435 were slightly less stable (Fig. S6). Thus, the simulations of single-point mutants of PYK2 did not provide clear indicators of differences in the structural behaviour of FAK and PYK2.

We next investigated whether the helix-like conformation of the DFG-motif region would be unfavourable in apo-PYK2. For this purpose, we built a "chimeric" PYK2 structure with the helical DFG motif region 
mirroring the one observed in FAK complexes with slowly dissociating compounds (see Methods section). Then, we performed a series of MD simulations ( $1 \mu \mathrm{s}$ in total) for FAK and PYK2 (both with the helical DFG-motif). The RMSD of all structural elements (the helical DFG-motif, $\beta 1$, and $\alpha \mathrm{C}$ ) of the binding site was quite low in the unbound FAK ( $<2 \AA$, Fig. S6), which indicated that the DFG-helical form in FAK was relatively stable even in the absence of a ligand. Surprisingly, the hydrogen-bond between G566 and Q432 was not preserved and appeared on average in only half of the frames (Fig. 6E). Thus, the presence of Q432 in FAK instead of F435 in PYK2 - while important - may not be the only factor that determines the DFG-motif conformation.

In contrast to FAK, the binding site in the "chimeric" structure of PYK2 was flexible and underwent considerable reconstruction (backbone RMSD of about $3 \AA$ for the helical segment containing the DFGmotif, Fig. S6). Specifically, the $\alpha \mathrm{C}$ moved away from the ATP binding pocket towards a position similar to that in the FAK structure (Fig. S6) and $\beta 1$ slightly changed its conformation. However, its first strand did not adopt the conformation observed in FAK, i.e. distorted in the middle (PYK2 and FAK are compared in Fig. 6C). Instead, it preserved the well-defined $\beta$-sheet structure of the original PYK2 conformation. As mentioned above, the difference in the $\beta$-sheet structure in PYK2 and FAK is likely caused by two residues: G425 and C427 in FAK are replaced by larger residues, N428 and 1430 in PYK2, respectively. As a result, in the "chimeric" PYK2 structure, $\beta 1$ and the following P-loop were shifted towards the helix of the DFGmotif and the following A-loop region by about $4 \AA$. This shift lead to the repositioning of $L 570$ as shown in Fig. 6C, where FAK bound to compound $\mathbf{1}$ is compared with the "chimeric" PYK2 structure after about 200 ns of equilibration. Finally, hydrogen-bonds between the helical DFG-motif fragment and the rest of the protein were different in "chimeric" PYK2 relative to FAK. Apart from the missing interaction with G569 (hydrogen-bond between G566 and R432 in FAK), the interactions of S571 and D572 were also less stable in PYK2 (Fig. 6E). All these results clearly indicate that, even with the same helical DFG-motif, the apostructure and stability of the binding site in PYK2 differs from FAK.

To assess the effect of the helical conformation of the DFG-motif loop on the ligand unbinding rate, we modelled compound 3 bound to the "chimeric" PYK2 structure in the same pose as in FAK (PDB 3bz3; Fig. S6, structures 3 and 4) and computed its residence time. The computed residence time of compound $\mathbf{3}$ was doubled relative to that for the loop DFG-motif conformation (see point denoted by "(3)" in Fig.4E) but it was still $\sim 5$-fold shorter than for the same compound bound to FAK. The difference between the protein-ligand interaction fingerprints of FAK and PYK2 is shown in Fig. 6D. Although the hydrogen bond between the sulfonamide moiety of the ligand and D569, as well as the hydrophobic interaction with L570, was still preserved in the complex with the "chimeric" PYK2 structure, some interactions with $\beta$-strands 
and the hinge loop (such as 1428, V436, A452, V484, L501) lining the pocket entrance were less stable than in FAK. This suggested that the protein-ligand interactions, particularly with $\beta 1-\beta 4$, were less optimal in PYK2 relative to FAK even if the helical conformation of the DFG-motif was present in both structures.

We further explored the effect of the structural differences of the PYK2 and FAK binding sites by making 12 constructs with point mutations and subsequently performing NanoBRET target engagement assays and wash-out experiments (Fig. 6F-H, Fig. S5, Table S3). First, we tested the interaction of the inhibitors with FAK-L567 by mutating this residue to an alanine and hypothesized a change from slow to fast off-rate as the missing hydrophobic interaction with the FAK protein. Indeed, the binding affinity of the inhibitor series was lower for FAK L567A and, gratifyingly, the mutation changed the off-rates from slow to fast, indicating a direct correlation of this residue and helix formation with dissociation kinetics (Fig. 6G). For testing the role of the polar interaction of the oxindole and quinoline-2-ones with FAK R426, we mutated it to alanine. In agreement with computational and crystallographic analysis, the potency did not change for the FAK R426A mutant and we did not observe differences in the binding kinetics (Fig. S5, Table S3).

In the crystal structure, we observed a hydrogen bond between PYK2 E509 and the pyridine nitrogen of 1 (Fig. 1) but this was not present for the equivalent FAK E506. In order to try to establish an interaction between the ligand aromatic ring system and FAK, we mutated FAK E506 to glutamine, to test for an electron donor, and to isoleucine to test for a hydrophobic interaction at this position. Both FAK E506Q and FAK E506I showed decreased potency for the series of inhibitors, indicating this interaction to be unfavourable in FAK (Fig. S5, Table S3). The P-loop of PYK2 is stabilized by a polar interaction of E432 sidechain (PYK2) with the backbone of G437. In FAK, the corresponding residue E430 pointed towards the solvent and did not contribute to a stabilized P-loop. Using FAK-E430S and FAK-E430Q mutants, we investigated whether we can establish the same P-loop rigidity as in PYK2, preventing the DFG helix from forming. However, the mutant binding kinetics were similar to the wildtype FAK binding kinetics (Fig. S5, Table S3). This result is also in accordance with the MD simulations showing that the hydrogen bond between G566 and R432 was not strictly preserved. As we see a striking difference in the sequence of FAK and PYK2 in PYK2-F435 (vs FAK Q432), we tested the PYK2 mutants F435Q and F435R. While the mutants did not show a change in potency, we did observe a change to slower binding kinetics for PYK2 F435Q. As E474 is located in helix $\alpha \mathrm{C}$ in PYK2, rigidifies the protein, we mutated E474 to glutamine, which resulted in a decreased potency but similar binding kinetics to WT PYK2 (Fig. S5, Table S3).

Lastly, we tested a potential involvement of protein phosphorylation in binding and kinetics by mutating the respective Y576 and Y577 in FAK and Y579 and Y580 in PYK2 to phenylalanine. While in FAK we did not observe a change in potency, the difference compared to PYK2 wild-type was a 2-fold increase in potency. 
However, these mutations had no impact on binding kinetics. In summary, for PYK2, none of the mutants showed a change from fast to slow dissociation for this series of inhibitors. As a representative for the inhibitor set, we chose 10, where only fast kinetics was observed for all PYK2 mutants (Fig. 6H). Based on both the mutagenesis study and our MD simulations, we concluded that it is not one single amino acid residue change that enables FAK to form a stable helical DFG-motif in complexes.

\section{Significance}

In this study, we explored the structural mechanism of amino-pyrimidine inhibitor binding, confirming existing FAK SARs and providing a SKR study for FAK. We observed slow-binding inhibitors to induce a helical DFG motif conformation in FAK and showed that the gained selectivity of inhibitor binding to FAK and PYK2 was based on a change in their unbinding rates driven by several sequence dissimilarities in their binding sites. This study demonstrates that small variations in the sequence of the protein binding site may trigger different mechanisms of ligand-protein interactions and result in ligand-induced stabilization of distinct protein conformations that affect ligand binding kinetics. We demonstrated that a combination of structural data and in silico methods can be used to predict compound residence times and to obtain insight into the molecular determinants affecting kinetics of inhibitors. We have shown here that the NanoBRET wash-out technique provides a unique opportunity to explore target engagement and kinetic behavior in living cells. The kinetic data translated into the in cellulo context of a NanoBRET wash-out assay and validated the PF-431396 (10) SPR based residence time of 138 minutes in living cells. Nevertheless, this cellular residence time of PF-431396 (10) was still short to significantly affect the pharmacology of 10 indicating that future drug discovery efforts are necessary to optimize ligand kinetics in order to impact clinical efficacy. Indeed, this amino-pyrimidine-series was developed into a macrocyclic inhibitor that showed better selectivity for PYK2 (Farand et al., 2016). The hydrophobic interaction of inhibitors with the helical activation loop in FAK should be included in future optimization processes for the development of inhibitors showing prolonged residence time for FAK.

\section{Acknowledgements}

Funding: This work was supported by a Capes-Humboldt postdoctoral scholarship to A N-A (Capes process number 88881.162167/2017-01), the European Union's Horizon 2020 Framework Programme for Research and Innovation under the Specific Grant Agreement No. 785907 and No. 945539 (Human Brain 
Project SGA2 and SGA3 to RCW), EU/EFPIA Innovative Medicines Initiative (IMI) Joint Undertaking, K4DD (grant no. 115366) and the Klaus Tschira Foundation. BTB, MS, JME, SMK and SK are supported by the Innovative Medicines Initiative 2 Joint Undertaking (JU) under grant agreement No 875510 and the SGC, a registered charity (number 1097737) that receives funds from; AbbVie, Bayer AG, Boehringer Ingelheim, Canada Foundation for Innovation, Eshelman Institute for Innovation, Genentech, Genome Canada through Ontario Genomics Institute [OGI-196], EU/EFPIA/OICR/McGill/KTH/Diamond, Innovative Medicines Initiative 2 Joint Undertaking [EUbOPEN grant 875510], Janssen, Merck KGaA (aka EMD in Canada and US), Merck \& Co (aka MSD outside Canada and US), Pfizer, São Paulo Research FoundationFAPESP, Takeda and Wellcome. B-T., B and S.K. would like to acknowledge funding by the DFG funded CRC SFB 1399.

\section{Author Contributions}

S.K., S.M., M.F., J.B., J.M.E., and R.W. supervised the research. T.H. synthesized the inhibitors. B-T.B, M.S., D.M., and M.A. performed structural studies. B-T. B, M.A., I.N., and J.W. performed biochemical and biophysical binding studies. B-T. B and M.A. performed NanoBRET cell-based ligand studies. D.B.K, A.N-A. and R.W. performed in silico analysis. S.K. M.A., D.B.K. and B-T. B drafted the manuscript, which has been edited and approved by all authors.

\section{Declaration of interests}

M.F. J.B. and T.H. are employees of Merck KGaA, Darmstadt, Germany. M.A. was an employee of Merck KGaA, Darmstadt, Germany at the time of experimental procedures and is now an employee of SanofiAventis Deutschland GmbH, Frankfurt, Germany. Other authors have no conflict of interest to declare.

\section{Main figure titles and legends}

Fig. 1: Comparison of the binding modes of PF-562271 (1) in FAK (PDB 3bz3 (Roberts et al., 2008)) and PYK2 (PDB 5tob (Farand et al., 2016)). A) Chemical structure of PF-562271 (1) and complexes of FAK (cyan) and PYK2 (grey) with PF-562271 (1, yellow). Kinase residues interacting with the inhibitor are highlighted in blue (detailed analysis in Table S1), hydrogen bonds are indicated by dashes. The DFG motif is depicted in orange for both kinases. Analysis of the interface between FAK and PF562271 (B, blue) and PYK2 and PF562271 (C, gray). Shown is the Buried Surface Area (BSA) normalized by the Accessible Surface Area (ASA) per residue. Compound structure was drawn using Biovia Draw 2017, models were depicted using PyMol 1.7.6.2 and bar charts were created using GraphPad Prism 8. 
Fig. 2: L567-ligand interactions increased potency and prolonged residence times in FAK. Slow off-rate inhibitors induce a helical conformation of the activation loop in FAK. A-F: The top of each panel shows the chemical structure of compounds $(\mathbf{2}, \mathbf{3}, \mathbf{4}, \mathbf{5}, \mathbf{8}, \mathbf{1 0})$ and their respective residence times $\tau$ of the inhibitor-FAK complexes. Shown below are the determined inhibitor bound FAK structures (FAK (cyan), inhibitors (yellow)) with highlighted DFG segment in orange. Dashes indicate hydrogen bonds. On the bottom of each panel amino acid residues contributing to the interface between FAK and the inhibitor are shown. Displayed is the Buried Surface Area (BSA) normalized by the Accessible Surface Area (ASA) per residue (detailed analysis in Table S1). PDB codes: 6yt6 (2), 6yxv (3), 6yq1 (4), 6yoj (5), 6yvs (8), 6yr9 (10). Compound structure was drawn using Biovia Draw 2017, models were depicted using PyMol 1.7.6.2 and bar charts were created using GraphPad Prism 8.

Fig. 3: The selectivity profile of FAK and PYK2 is dictated by the formation of a helical conformation of the DFG motif in FAK. A) Overlay of crystal structures of the complexes of FAK-10 (blue and yellow, PDB code: 6yr9) and PYK2-10 (gray and magenta, PDB code: 3 fzr (Han et al., 2009)), B) Overlay of the complexes of FAK-12 (blue and yellow, PDB code: 6yvy) and PYK2-12 (gray and magenta, PDB code: 3h3c (Walker et al., 2009)). Models were depicted using PyMol 1.7.6.2.

Fig. 4: Protein flexibility and the dynamics of the protein-ligand interactions differ for FAK and PYK2. The analysis of MD simulations of complexes of ligands with PYK2 and FAK is shown along with computed relative residence times: A) RMSF of the ligand and three regions of the binding site ( $\beta 1, \alpha C$ and the DFG loop corresponding, respectively, to FAK residues 421-434 , 451-471, and 563-570) averaged over four MD trajectories (80ns in total) and shown for 12 compounds for FAK and PYK2. B, C) Protein-ligand interaction fingerprint maps for ligand-protein complexes obtained from the last 300 frames of four 20ns conventional MD simulations for FAK B) and PYK2 C). The occurrence of hydrogen-bonds (HD/HA), aromatic (AR), hydrophobic (HY), and water-mediated (WB) protein-ligand interactions is shown by the colour (white not observed, dark blue - present in all snapshots analysed). The SPR residence times are shown on a logarithmic scale for all compounds in the right-hand plots. D) View of compound $\mathbf{1}$ and residues in FAK that contribute to the protein-ligand interactions shown in B). E) Correlation between computed relative residence times ( $\tau_{\text {RAMD }}$ ) and $1 / k_{\text {off }}$ values measured by SPR for FAK and PYK2; $R^{2}=0.91$ for the entire set of the data ( $R^{2}=0.95$ and 0.63 for the FAK and PYK2 subsets, respectively). Compound 3 simulated starting from a complex with a helical conformation of the DFG-motif in PYK2 is denoted by "(3)" (see text and Fig. S4A). Figures were created using Python (library Matplotlib) and models were depicted using PyMol 1.7.6.2.

Fig. 5: Binding potency and kinetics of FAK and PYK2 in living cells. A) Target engagement assays for FAK and PYK2 for 1 and $\mathbf{1 0}$ as indicated in the figure legend. B) Correlation of the in cellulo potency with the potency determined in vitro by SPR. C) NanoBRET wash-out experiments for $\mathbf{1}$ and $\mathbf{1 0}$ in comparison to background data and tracer only (DMSO added instead of test compound). Figures were created using GraphPad Prism 8.

Fig. 6: Structural stability of the ligand binding sites in FAK and PYK2 explored by computations and by experimental site-directed mutagenesis: (A-E) Computations: (A,B) Binding site in FAK (left, PDB ID 3bz3) and PYK2 (right, PDB ID 3et7) indicating residues that may affect protein flexibility (see discussion in the main text) in stick representation; (C, E) Results of simulations of unbound FAK and "chimeric" PYK2 structures with a helical conformation of the DFG-motif: (C) Variation of the structures of FAK (grey) and chimeric PYK2 (beige) in conventional MD simulations (four frames are shown for each protein); $L 570$ in PYK2 is highlighted in orange, the ligand position in a FAK complex is shown for compound $\mathbf{1}$ (PDB ID 3bz3); (E) Fraction of MD frames where hydrogen bonds between six residues of the DFG-motif helix and the rest of the protein were observed; (D): Protein-ligand interaction fingerprint map for compound $\mathbf{3}$ bound to 
FAK and PYK2 ( with helical or loop conformation of the DFG motif (see Fig.4B for key); residues are labelled by the name and number in the FAK structure; $(\mathrm{F}-\mathrm{H})$ : Site directed mutagenesis: $(\mathrm{F})$ NanoBRET potency for mutant constructs displayed as a ratio of $\mathrm{plC}_{50}$ Mutant / Wild-Type for mutants as indicated in the figure. (G-H) Representative wash-out experiment for $\mathbf{1 0}$ on the mutants tested for FAK (G) and PYK2 (H). The signal was normalised to the tracer control and fitted using a one phase exponential decay. Models A$\mathrm{C}$ were created using PyMol 1.7.6.2, Figures D-E and figures F-H were created using Python (library Matplotlib) and GraphPad Prism 8, respectivly. 
Main tables and legends

\begin{tabular}{|c|c|c|c|c|c|c|c|c|c|}
\hline \multirow[b]{2}{*}{$\begin{array}{l}\text { Cmpd No } \\
\text { (Name) }\end{array}$} & & & & \multicolumn{3}{|c|}{ FAK (SPR) } & \multicolumn{3}{|c|}{ PYK2 (SPR) } \\
\hline & $\mathbf{R}^{1}$ & $\mathbf{R}^{2}$ & $\mathbf{R}^{3}$ & $\begin{array}{c}k_{\text {on }} \\
{\left[10^{5} / \mathrm{Ms}\right]}\end{array}$ & $\begin{array}{c}k_{\text {off }} \\
{\left[10^{-2} / s\right]}\end{array}$ & $\begin{array}{c}K_{\mathrm{D}} \\
{[\mathrm{nM}]}\end{array}$ & $\begin{array}{c}k_{\mathrm{on}} \\
{\left[10^{5} / \mathrm{Ms}\right]}\end{array}$ & $\begin{array}{c}k_{\text {off }} \\
{\left[10^{-2} / s\right]}\end{array}$ & $\begin{array}{c}K_{\mathrm{D}} \\
{[\mathrm{nM}]}\end{array}$ \\
\hline $\begin{array}{l}1 \\
\text { PF-562271 }\end{array}$ & & & $-\mathrm{CF}_{3}$ & $53 \pm 20.0$ & $0.34 \pm 0.078$ & $0.76 \pm 0.35$ & $130 \pm 43$ & $7.4 \pm 2.5$ & $5.7 \pm 1.0$ \\
\hline 2 & & & $-H$ & $8.8 \pm 2.4$ & $29 \pm 8.8$ & $320 \pm 27$ & $9.0 \pm 3.3$ & $75 \pm 32$ & $820 \pm 96$ \\
\hline 3 & & & $-\mathrm{CF}_{3}$ & $0.87 \pm 0.22$ & $0.62 \pm 0.24$ & $70 \pm 22$ & $0.37 \pm 0.15$ & $\begin{array}{c}1.7 \pm 0.7 \\
8\end{array}$ & $470 \pm 100$ \\
\hline 4 & & & $-\mathrm{CF}_{3}$ & n.d. & n.d. & n.d. & n.d. & n.d. & n.d. \\
\hline $\begin{array}{l}5 \\
\text { PF-573228 }\end{array}$ & & & $-\mathrm{CF}_{3}$ & $70.2 \pm 36$ & $0.82 \pm 0.22$ & $1.3 \pm 0,32$ & $13 \pm 4.8$ & $33 \pm 11$ & $250 \pm 19$ \\
\hline 6 & & & $-\mathrm{CF}_{3}$ & $17 \pm 10$ & $6.1 \pm 4.2$ & $37 \pm 7.0$ & $17 \pm 6.6$ & $19 \pm 7.8$ & $110 \pm 4.0$ \\
\hline 7 & & & $-\mathrm{CF}_{3}$ & $1.4 \pm 0.44$ & $14 \pm 3.0$ & $1000 \pm 23$ & \multicolumn{3}{|c|}{ No binding } \\
\hline 8 & & & $-\mathrm{CF}_{3}$ & $48 \pm 20$ & $7.1 \pm 3.0$ & $15 \pm 2.6$ & $13 \pm 8.4$ & $25 \pm 11$ & $230 \pm 92$ \\
\hline 9 & & & $-\mathrm{CF}_{3}$ & $1.8 \pm 0.51$ & $4.8 \pm 1.3$ & $260 \pm 21$ & \multicolumn{3}{|c|}{ No binding } \\
\hline $\begin{array}{l}10 \\
\text { PF-431396 }\end{array}$ & & & $-\mathrm{CF}_{3}$ & $30.8 \pm 14$ & $0.054 \pm 0.027$ & $0.18 \pm 0.05$ & $58 \pm 15$ & $4.0 \pm 1.2$ & $6.9 \pm 0.54$ \\
\hline $\begin{array}{l}11 \\
\text { PF-8554878 } \\
\text { defactinib }\end{array}$ & & & $-\mathrm{CF}_{3}$ & $24 \pm 5.8$ & $0.44 \pm 0.061$ & $1.9 \pm 0.50$ & $15 \pm 3.8$ & $3.6 \pm 1.1$ & $24 \pm 3.1$ \\
\hline $\begin{array}{l}12 \\
\text { PF-719 }\end{array}$ & & & $-\mathrm{CF}_{3}$ & $14 \pm 6.5$ & $20 \pm 8.3$ & $150 \pm 19$ & $22 \pm 8.1$ & $5.5 \pm 2.4$ & $25 \pm 3.3$ \\
\hline
\end{tabular}

Table 1: Design of a series of inhibitors probing the structure activity relationship in FAK and PYK2. Three substituent sites on the pyrimidine core structure of $1, R^{1}$ (solvent exposed), $R^{2}$ (DFG adjacent) and $R^{3}$ (back pocket) were considered to study the interactions in the kinase binding sites. SPR= Surface Plasmon Resonance, $k_{\mathrm{on}}=$ association rate constant, $k_{\text {off }}=$ dissociation rate constant, $k_{\mathrm{D}}=$ equilibrium dissociation constant, n.d.= not determined. 


\section{STAR*Methods}

- RESOURCE AVAILABILITY

- Lead Contact

- Materials Availability

- Data and code availability

- EXPERIMENTAL MODEL AND SUBJECT DETAILS

○ Microbe strains

- Cell lines

- METHOD DETAILS

- Compound Chemistry

- Protein Expression and Purification

- Crystallization and Structure Determination

- Surface Plasmon Resonance

- NanoBRET cellular assays

- NanoBRET plasmid mutation

- Computations

- Computational preparation of structures

- Docking of compounds to PYK2

- Modeling of PYK with a FAK-like DFG helix

- MD and $\tau$ RAMD simulations

- Analysis of MD trajectories

- QUANTIFICATION AND STATISTICAL ANALYSIS

- Surface-Plasmon-Resonance-Data

- NanoBRET Data

RESOURCE AVAILABILITY

Lead Contact

Further information and requests for resources and reagents should be directed to and will be fulfilled by the Lead Contact, Stefan Knapp (knapp@pharmchem.uni-frankfurt.de).

\section{Material Availability}

Requests for plasmids generated in this study should be directed to the lead contact. Inhibitors used in this study can be obtained from commercial sources or for inhibitors generated in this study requests can be directed to Timo Heinrich.

\section{Data and code availability}

The coordinates and structure factors of all complexes have been deposited to the protein data bank under accession codes: 6YT6, 6YXV, 6YQ1, 6YOJ, 6YVS, 6YR9, 6YVY. 


\section{Experimental Model and Subject Details}

\section{Microbe strains}

Escherichia coli, BL21 (DE3) strain was used for recombinant protein expression. Cells were cultured in terrific broth media at $37^{\circ} \mathrm{C}$ until they reached an OD600 of 2.6-3.0 prior to induction with $0.5 \mathrm{mM}$ IPTG for protein expression at $18^{\circ} \mathrm{C}$ overnight.

\section{Cell lines}

HEK293T (female) cells were obtained from ATCC (identifier CRL-3216). Cells were cultured at $37^{\circ} \mathrm{C} / 5 \%$ $\mathrm{CO}_{2}$ in DMEM (Gibco) supplemented with 10\% heat inactivated FBS (Invitrogen) and penicillin-streptavidin antibiotic (Gibco).

\section{Method Details}

\section{Compound Chemistry.}

\section{General synthesis of diaminopyrimidines $1-6,8,10-12$}

The synthetic steps were performed as described in the patent literature (Arcari et al., 2006; Kath and Luzzio, 2003; Luzzio et al., 2008; Matsuhisa et al., 2001) and are described in detail below.

Synthesis of N-Methyl-N-(3-\{[2-(2-oxo-2,3-dihydro-1H-indol-5-ylamino)-5-trifluoromethyl-pyrimidin-4ylamino]-methyl\}-pyridin-2-yl)-methanesulfonamide (PF- 562271) (1)

The reaction of 5-trifluoromethyl-2,4-dichloropyrimidine and 5-amino-oxindole in a tert butanol/dichloroethane $=1: 1$ and zinc chloride yielded the intermediate 5-\{[4-chloro-5(trifluoromethyl)pyrimidin-2-yl]amino\}-2,3-dihydro-1H-indol-2-one with 59\%. This compound reacted with $\mathrm{N}$-[3-(aminomethyl)pyridin-2-yl]-N-methylmethanesulfonamide dihydrochloride in DMF and 2.5 eq of sodium carbonate at $85^{\circ} \mathrm{C}$ over night to the final product in $36 \%$ yield. The yield for the final step was optimized by reaction in toluene/isopropanol = 1:1 in the presence of 1.2 eq of diisopropylethylamine to $72 \%$.

${ }^{1} \mathrm{H}$ NMR $\left(500 \mathrm{MHz}, \mathrm{DMSO}-d_{6}\right) \delta 10.16(\mathrm{~s}, 2 \mathrm{H}), 9.37(\mathrm{~s}, 1 \mathrm{H}), 8.44(\mathrm{dd}, J=4.7,1.8 \mathrm{~Hz}, 3 \mathrm{H}), 8.21(\mathrm{~s}, 3 \mathrm{H}), 7.67$ $(\mathrm{d}, J=8.0 \mathrm{~Hz}, 3 \mathrm{H}), 7.50-7.44(\mathrm{~m}, 3 \mathrm{H}), 7.42(\mathrm{dd}, J=7.8,4.7 \mathrm{~Hz}, 3 \mathrm{H}), 7.33(\mathrm{~s}, 2 \mathrm{H}), 7.19(\mathrm{~d}, J=8.3 \mathrm{~Hz}, 2 \mathrm{H})$, $6.54(\mathrm{~d}, J=8.3 \mathrm{~Hz}, 2 \mathrm{H}), 4.77(\mathrm{~d}, J=5.8 \mathrm{~Hz}, 5 \mathrm{H}), 4.03(\mathrm{q}, J=7.1 \mathrm{~Hz}, 2 \mathrm{H}), 3.15(\mathrm{~s}, 9 \mathrm{H}), 3.11(\mathrm{~s}, 7 \mathrm{H}), 1.99(\mathrm{~s}$, $3 \mathrm{H}), 1.18(\mathrm{t}, J=7.1 \mathrm{~Hz}, 3 \mathrm{H})$.

Synthesis of $\mathrm{N}$-Methyl-N-(3-\{[2-(2-oxo-2,3-dihydro-1H-indol-5-ylamino)-pyrimidin-4-ylamino]-methyl\}pyridin-2-yl)-methanesulfonamide (2) 
methylmethanesulfonamide was obtained in $27 \%$ yield by reaction of 2,4-dichloropyrimidine and $\mathrm{N}$-[3(aminomethyl)pyridin-2-yl]-N-methylmethanesulfonamide in the microwave at $120^{\circ} \mathrm{C}$ for 20 minutes in the presence of 2 eq of triethyl amine. The final product was obtained after reaction of the intermediate with 5 -aminoindole under identical conditions in $30 \%$ yield as brown solid.

${ }^{1} \mathrm{H}$ NMR $\left(500 \mathrm{MHz}, \mathrm{DMSO}-d_{6}\right) \delta 10.11(\mathrm{~s}, 2 \mathrm{H}), 8.76(\mathrm{~s}, 2 \mathrm{H}), 8.45(\mathrm{dd}, J=4.7,1.9 \mathrm{~Hz}, 2 \mathrm{H}), 8.15(\mathrm{~s}, 3 \mathrm{H}), 7.85$ - $7.79(\mathrm{~m}, 4 \mathrm{H}), 7.56(\mathrm{~s}, 2 \mathrm{H}), 7.52(\mathrm{~s}, 2 \mathrm{H}), 7.44(\mathrm{dd}, J=7.7,4.7 \mathrm{~Hz}, 2 \mathrm{H}), 7.39-7.33(\mathrm{~m}, 2 \mathrm{H}), 6.59(\mathrm{~d}, J=8.4$ $\mathrm{Hz}, 2 \mathrm{H}), 5.99-5.95(\mathrm{~m}, 1 \mathrm{H}), 4.65(\mathrm{~d}, J=5.9 \mathrm{~Hz}, 3 \mathrm{H}), 3.17(\mathrm{~d}, J=7.1 \mathrm{~Hz}, 13 \mathrm{H})$.

Synthesis of N-Methyl-N-\{3-[(2-phenylamino-5-trifluoromethyl-pyrimidin-4-ylamino)-methyl]-pyridin-2-yl\}methanesulfonamide (3)

The intermediate 4-chloro-N-phenyl-5-(trifluoromethyl)pyrimidin-2-amine was obtained in $58 \%$ yield by reaction of 5-trifluoromethyl-2,4-dichloropyrimidine and aniline in dichloromethane/tert-butanol = 1:1 in the presence of stochiometric amounts of zing chloride-diethylether complex and triethyl amine at RT over night. The final product could be isolated in $40 \%$ yield by reaction of the intermediate with $\mathrm{N}$-[3(aminomethyl)pyridin-2-yl]-N-methylmethanesulfonamide in toluene/isopropanol $=1: 1$ at $80^{\circ} \mathrm{C}$ over night and column chromatography.

${ }^{1} \mathrm{H}$ NMR $\left(400 \mathrm{MHz}, \mathrm{DMSO}-d_{6}\right) \delta 9.54(\mathrm{~s}, 1 \mathrm{H}), 8.44(\mathrm{dd}, J=4.7,1.9 \mathrm{~Hz}, 1 \mathrm{H}), 8.26(\mathrm{~s}, 1 \mathrm{H}), 7.66(\mathrm{dd}, J=7.7$, $1.9 \mathrm{~Hz}, 1 \mathrm{H}), 7.58(\mathrm{t}, J=5.9 \mathrm{~Hz}, 1 \mathrm{H}), 7.42(\mathrm{dt}, J=7.9,4.5 \mathrm{~Hz}, 3 \mathrm{H}), 7.08(\mathrm{t}, J=7.7 \mathrm{~Hz}, 2 \mathrm{H}), 6.88(\mathrm{t}, J=7.3 \mathrm{~Hz}$, $1 \mathrm{H}), 4.81(\mathrm{~d}, J=5.7 \mathrm{~Hz}, 2 \mathrm{H}), 3.15(\mathrm{~d}, J=11.9 \mathrm{~Hz}, 6 \mathrm{H})$.

Synthesis of N-Methyl-N-(3-\{[2-(2-oxo-1,2,3,4-tetrahydro-quinolin-6-ylamino)-5-trifluoromethyl-pyrimidin4-ylamino]-methyl\}-pyridin-2-yl)-methanesulfonamide (4)

Reaction of 5-drifluoromethyl-2,4-dichloropyrimidine and 6-amino-3,4-dihydroquinolin-2(1H)-one in tert butanol/dichloromethane in the presence of zinc chloride gave the intermediate 6-(4-Chloro-5(trifluoromethyl)pyrimidine-2-ylamino-3,4-dihydoquinoline-2(1H)-one in $64 \%$ yield. This compound reacted to the final product in $31 \%$ yield with $\mathrm{N}$-[3-(aminomethyl)pyridin-2-yl]-Nmethylmethanesulfonamide.

${ }^{1} \mathrm{H}$ NMR (500 MHz, DMSO-d6) d $9.89(\mathrm{~s}, 1 \mathrm{H}), 9.45-9.33(\mathrm{~m}, 1 \mathrm{H}), 8.43(\mathrm{dd}, \mathrm{J}=4.8,1.8 \mathrm{~Hz}, 1 \mathrm{H}), 8.23-8.21$ $(\mathrm{m}, 1 \mathrm{H}), 7.68(\mathrm{~d}, \mathrm{~J}=7.7 \mathrm{~Hz}, 1 \mathrm{H}), 7.49(\mathrm{t}, \mathrm{J}=5.8 \mathrm{~Hz}, 1 \mathrm{H}), 7.41(\mathrm{dd}, \mathrm{J}=7.8,4.7 \mathrm{~Hz}, 1 \mathrm{H}), 7.32-7.26(\mathrm{~m}, 1 \mathrm{H})$, $7.19(\mathrm{~d}, \mathrm{~J}=8.5 \mathrm{~Hz}, 1 \mathrm{H}), 6.60(\mathrm{~d}, \mathrm{~J}=8.6 \mathrm{~Hz}, 1 \mathrm{H}), 4.79(\mathrm{~d}, \mathrm{~J}=5.7 \mathrm{~Hz}, 2 \mathrm{H}), 3.14(\mathrm{~s}, 3 \mathrm{H}), 3.14-3.11(\mathrm{~m}, 3 \mathrm{H})$, $2.66-2.57(\mathrm{~m}, 2 \mathrm{H}), 2.36-2.31(\mathrm{~m}, 2 \mathrm{H})$. 
Synthesis of 6-[4-(3-Methanesulfonyl-benzylamino)-5-trifluoromethyl-pyrimidin-2-ylamino]-3,4-dihydro1H-quinolin-2-one (PF-573228) (5)

The intermediate from the preparation of compound 4 reacted with (3-(methylsulfonyl)phenyl) methanamine $\mathrm{HCl}$ salt in NMP in the presence of 3 eq of triethylamine to the final compound in $70 \%$ yield.

${ }^{1} \mathrm{H}$ NMR $\left(400 \mathrm{MHz}\right.$, DMSO-d $\left.d_{6}\right) \delta 9.89(\mathrm{~s}, 1 \mathrm{H}), 9.44$ (br. s, 1H), $8.16(\mathrm{~s}, 1 \mathrm{H}), 7.83-7.76(\mathrm{~m}, 3 \mathrm{H}), 7.56-7.54$ $(\mathrm{m}, 2 \mathrm{H}), 7.34(\mathrm{~s}, 1 \mathrm{H}), 7.19(\mathrm{~d}, J=7.3 \mathrm{~Hz}, 1 \mathrm{H}), 6.64(\mathrm{~d}, J=8.7 \mathrm{~Hz}, 1 \mathrm{H}), 4.70(\mathrm{~d}, J=5.5 \mathrm{~Hz}, 2 \mathrm{H}), 3.34(\mathrm{~s}, 3 \mathrm{H})$, $2.63(\mathrm{t}, J=7.3 \mathrm{~Hz}, 2 \mathrm{H}), 2.34(\mathrm{t}, J=11 \mathrm{~Hz}, 2 \mathrm{H})$.

Synthesis of 5-(\{4-[(2-oxo-2,3-dihydro-1H-indol-5-yl)amino]-5-(trifluoromethyl)pyrimidin-2-yl\}amino)-2,3dihydro-1H-indol-2-one (6)

The intermediate 5-\{[4-chloro-5-(trifluoromethyl)pyrimidin-2-yl]amino\}-2,3-dihydro-1H-indol-2-one obtained for the synthesis of $\mathbf{8}$ was repeated with an access of 5-amino-oxindole and the final product was isolated in $77 \%$ after chromatography.

${ }^{1} \mathrm{H}$ NMR $\left(400 \mathrm{MHz}, \mathrm{DMSO}-d_{6}\right) \delta 10.44(\mathrm{~s}, 2 \mathrm{H}), 10.16(\mathrm{~s}, 2 \mathrm{H}), 9.47(\mathrm{~s}, 1 \mathrm{H}), 8.56(\mathrm{~s}, 1 \mathrm{H}), 8.26(\mathrm{~s}, 2 \mathrm{H}), 7.43(\mathrm{~s}$, $2 \mathrm{H}), 7.24-7.16(\mathrm{~m}, 6 \mathrm{H}), 6.84(\mathrm{~d}, J=8.1 \mathrm{~Hz}, 2 \mathrm{H}), 6.55(\mathrm{~d}, J=8.4 \mathrm{~Hz}, 2 \mathrm{H}), 3.47(\mathrm{~s}, 4 \mathrm{H})$.

Synthesis of 5-\{4-[(Pyridin-3-ylmethyl)-amino]-5-trifluoromethyl-pyrimidin-2-ylamino\}-1,3-dihydro-indol-2one (8)

To solution of 5-trifluoromethyl-2,4-dichloropyrimidine in 1:1 DCE/tBuOH was added Zinc chloride $1 \mathrm{M}$ solution in ether. After 0.5 hour, 5-amino-oxindole was added followed by triethylamine keeping temperature at $25^{\circ} \mathrm{C}$. The reaction was allowed to stir at room temperature overnight, then was concentrated and the product triturated (repeated several times, slightly soluble in $\mathrm{MeOH}$ ) from methanol as a yellow solid (5-\{[4-chloro-5-(trifluoromethyl)pyrimidin-2-yl]amino\}-2,3-dihydro-1H-indol-2-one, 65\% yield). This intermediate was dissolved in DMF and 1-(pyridin-3-yl)methanamine and sodium carbonate were added. The mixture reacted for 3 days at RT and the final product could be obtained after chromatography in $34 \%$ yield.

${ }^{1} \mathrm{H}$ NMR $\left(400 \mathrm{MHz}, \mathrm{DMSO}-d_{6}\right) \delta 10.21(\mathrm{~s}, 1 \mathrm{H}), 9.41(\mathrm{~s}, 1 \mathrm{H}), 8.53(\mathrm{~s}, 1 \mathrm{H}), 8.42(\mathrm{dd}, J=4.9,1.7 \mathrm{~Hz}, 1 \mathrm{H}), 8.17$ $(\mathrm{d}, J=5.3 \mathrm{~Hz}, 2 \mathrm{H}), 7.73(\mathrm{t}, J=6.0 \mathrm{~Hz}, 1 \mathrm{H}), 7.66(\mathrm{~d}, J=7.6 \mathrm{~Hz}, 1 \mathrm{H}), 7.38(\mathrm{~s}, 1 \mathrm{H}), 7.33(\mathrm{dd}, J=7.8,4.7 \mathrm{~Hz}$, $1 \mathrm{H}), 7.24(\mathrm{~d}, J=8.3 \mathrm{~Hz}, 1 \mathrm{H}), 6.65(\mathrm{~d}, J=8.4 \mathrm{~Hz}, 1 \mathrm{H}), 4.64(\mathrm{~d}, J=5.9 \mathrm{~Hz}, 2 \mathrm{H})$.

Synthesis of N-Methyl-N-(2-\{[2-(2-oxo-2,3-dihydro-1H-indol-5-ylamino)-5-trifluoromethyl-pyrimidin-4ylamino]-methyl\}-phenyl)-methanesulfonamide (PF-431396) (10) 
The intermediate 5-\{[4-chloro-5-(trifluoromethyl)pyrimidin-2-yl]amino\}-2,3-dihydro-1H-indol-2-one prepared for the synthesis of compound $\mathbf{8}$ was reacted with $\mathrm{N}$-[2-(aminomethyl)phenyl]-Nmethylmethanesulfonamide under identical conditions and yielded the title compound in $45 \%$ yield.

${ }^{1} \mathrm{H}$ NMR (400 MHz, DMSO) $\delta 10.24(\mathrm{~s}, 1 \mathrm{H}), 9.75$ (br. s, 1H), $8.26(\mathrm{~s}, 1 \mathrm{H}), 7.76$ (br. s, 1H), $7.56-7.53$ (m, $1 \mathrm{H}), 7.41-7.32(\mathrm{~m}, 3 \mathrm{H}), 7.27-7.22(\mathrm{~m}, 2 \mathrm{H}), 6.62-6.60(\mathrm{~m}, 1 \mathrm{H}), 4.81-4.79(\mathrm{~m}, 2 \mathrm{H}), 3.32-3.27(\mathrm{~m}, 2 \mathrm{H})$, $3.12-3.09(\mathrm{~m}, 6 \mathrm{H})$.

Synthesis of 4-(4-\{[3-(Methanesulfonyl-methyl-amino)-pyrazin-2-ylmethyl]-amino\}-5-trifluoromethylpyrimidin-2-ylamino)-N-methyl-benzamide (PF-04554878) (11)

The reaction of $\mathrm{N}$-(3-Aminomethyl-pyrazin-2-yl)-N-methyl-methanesulfonamide and 2,4-Dichloro-5trifluoromethyl-pyrimidine in dichloromethane in the presence of diisopropylethylamine from $-20^{\circ} \mathrm{C}$ to $0^{\circ} \mathrm{C}$ yielded the intermediate $\mathrm{N}$-\{3-[(2-Chloro-5-trifluoromethyl-pyrimidin-4-ylamino)-methyl]-pyrazin-2-yl\}-Nmethyl-methanesulfonamide in $32 \%$ yield. The final product was obtained by reaction of this intermediate with 4-amino- $\mathrm{N}$-methyl-benzamide in butanol and catalytic amounts of acetic acid from RT to $100^{\circ} \mathrm{C}$ in $14 \%$ yield.

${ }^{1} \mathrm{H}$ NMR $(400 \mathrm{MHz}, \mathrm{DMSO}) \delta 9.84(\mathrm{~s}, 1 \mathrm{H}), 8.68-8.67(\mathrm{~d}, \mathrm{~J}=4 \mathrm{~Hz}, 1 \mathrm{H}), 8.57-8.56(\mathrm{~d}, \mathrm{~J}=4 \mathrm{~Hz}, 1 \mathrm{H}), 8.30(\mathrm{~s}$, $1 \mathrm{H}), 8.20-8.19(\mathrm{~s}, \mathrm{~J}=4 \mathrm{~Hz}, 1 \mathrm{H}), 7.65-7.58(\mathrm{~m}, 3 \mathrm{H}), 7.43(\mathrm{~s}, 1 \mathrm{H}), 4.99-4.98(\mathrm{~d}, 2 \mathrm{H}), 3.21-3.18(\mathrm{~d}, 6 \mathrm{H}), 2.74-$ $2.73(\mathrm{~d}, 3 \mathrm{H})$

\section{General procedure for the preparation of aminopyrimidines $\mathbf{7}$ and $\mathbf{9}$.}

Intermediate 5-\{[4-chloro-5-(trifluoromethyl)pyrimidin-2-yl]amino\}-2,3-dihydro-1H-indol-2-one from the synthesis of compound $\mathbf{8}$ was coupled to the corresponding aryl acetylene in the presence of catalytic amounts of bistriphenylphosphine palladiumdichloride, copper(I)iodide and two equivalents of triethylamine in $\mathrm{DMF}$ at $100^{\circ} \mathrm{C}$ under $\mathrm{N}_{2}$ atmosphere to the corresponding diaryl-acetylene. The triple bond was reduced on $\mathrm{Pd} / \mathrm{C}$ contact at room temperature under an atmosphere of $\mathrm{H}_{2}$ for $\mathrm{h}$ in methanol.

Synthesis of 5-\{4-[2-(4-Fluoro-phenyl)-ethyl]-5-trifluoromethyl-pyrimidin-2-ylamino\}-1,3-dihydro-indol-2one (7)

The intermediate 5-(\{4-[2-(4-fluorophenyl)ethynyl]-5-(trifluoromethyl)pyrimidin-2-yl\}amino)-2,3-dihydro$1 \mathrm{H}$-indol-2-one was obtained in $92 \%$ and the final product in $75 \%$ yield.

${ }^{1} \mathrm{H}$ NMR (400 MHz, DMSO) $\delta 10.31(\mathrm{~s}, 1 \mathrm{H}), 10.07(\mathrm{~s}, 1 \mathrm{H}), 8.61(\mathrm{~s}, 1 \mathrm{H}), 7.58(\mathrm{~s}, 1 \mathrm{H}), 7.48(\mathrm{~d}, J=8.6 \mathrm{~Hz}, 1 \mathrm{H})$, $7.247 .24(\mathrm{dd}, J=8.2 \mathrm{~Hz}, 5.8 \mathrm{~Hz}, 2 \mathrm{H}), 7.10(\mathrm{t}, J=8.8 \mathrm{~Hz}, 2 \mathrm{H}), 6.76(\mathrm{~d}, J=8.4 \mathrm{~Hz}, 1 \mathrm{H}), 3.48(\mathrm{~s}, 2 \mathrm{H}), 3.13-$ $2.93(\mathrm{~m}, 4 \mathrm{H})$. 
Synthesis of 5-(4-Phenethyl-5-trifluoromethyl-pyrimidin-2-ylamino)-1,3-dihydro-indol-2-one (9).

The intermediate 5-\{[4-(2-phenylethynyl)-5-(trifluoromethyl)pyrimidin-2-yl]amino\}-2,3-dihydro-1H-indol2-one was obtained in $90 \%$ and the final product in $80 \%$ yield.

${ }^{1} \mathrm{H}$ NMR (400 MHz, DMSO) $\delta 10.31(\mathrm{~s}, 1 \mathrm{H}), 10.07(\mathrm{~s}, 1 \mathrm{H}), 8.61(\mathrm{~s}, 1 \mathrm{H}), 7.58(\mathrm{~s}, 1 \mathrm{H}), 7.48(\mathrm{~d}, J=8.6 \mathrm{~Hz}, 1 \mathrm{H})$, $7.29(\mathrm{t}, J=7.4 \mathrm{~Hz}, 2 \mathrm{H}), 7.20(\mathrm{dd}, J=15.2 \mathrm{~Hz}, 7.1 \mathrm{~Hz}, 3 \mathrm{H}), 6.76(\mathrm{~d}, J=8.4 \mathrm{~Hz}, 1 \mathrm{H}), 3.48(\mathrm{~s}, 2 \mathrm{H}), 3.03(\mathrm{~s}, 4 \mathrm{H})$.

\section{Protein Expression and Purification.}

Human FAK kinase domain was expressed and purified by Instituto de Biologia Experimental e Tecnológica. The FAK catalytic domain used for crystallization experiments and SPR assays was expressed with an $\mathrm{NH}_{2}$ terminal 6XHis-tag and comprises residues 410 to 689 after thrombin cleavage (FAK sequence with residue 410 changed from a Pro to a Gly). FAK was expressed in Hi5 insect cells using the Bac Magic kit (Invitrogen Corp.) Cells were harvested by centrifugation and resuspended in lysis buffer ( $20 \mathrm{mM} \mathrm{Na}-\mathrm{P}, 500 \mathrm{mM} \mathrm{NaCl}$, $0.1 \% \mathrm{NP}$ 40, $5 \mathrm{mM} \mathrm{MgCl}$, pH 7.5, $1 \mathrm{mM}$ DTT, benzonase and protease inhibitor cocktail III). Cells were lysed by high pressure homogenization and cleared by centrifugation at $31.000 \mathrm{xg}$ for $40 \mathrm{~min}$ at $4{ }^{\circ} \mathrm{C}$. The supernatant was loaded onto a Ni-NTA affinity column and the target protein was eluted in step gradient with buffer $20 \mathrm{mM}$ Na-P, $500 \mathrm{mM} \mathrm{NaCl}, 500$ mM Imidazole, 1 mM DTT, pH 7.5. Peak fractions were desalted and treated with hu-alpha Thrombin for histidine tag removal. The cleaved proteins were separated by passing through Ni-Sepharose resin and further purified using size-exclusion chromatography (Superdex $7526 / 60$ ). The resultant pure recombinant FAK was concentrated to $5.9 \mathrm{mg} / \mathrm{mL}$ and stored at $-80{ }^{\circ} \mathrm{C}$ in a buffer containing $10 \mathrm{mM}$ HEPES, $200 \mathrm{mM}$ ammonium sulfate, and 0.1 mM TCEP, pH 7.5.

The human PYK2 kinase domain used for SPR measurements was expressed with an $\mathrm{NH}_{2}$-terminal 6XHistag and comprised residues 420-691. PYK2 was cloned into pET28a including an N-terminal HIS-TEV-tag and expressed in Escherichia coli Rosetta 2 (DE3) cells. Cells were harvested by centrifugation and resuspended in lysis buffer ( $20 \mathrm{mM} \mathrm{Na}-\mathrm{P}, 500 \mathrm{mM} \mathrm{NaCl}, 1 \mathrm{mM}$ DTT, $20 \mathrm{mM}$ Imidazole, $5 \mathrm{mM} \mathrm{MgCl}$, pH 7.5, protease inhibitor cocktail III and Benzonase). Cells were lysed by high pressure homogenization and cleared by centrifugation at $20.400 \mathrm{xg}$ for $60 \mathrm{~min}$ at $4{ }^{\circ} \mathrm{C}$. Subsequent steps were similar to previously described for FAK. The resultant pure recombinant PYK2 was concentrated to $10.7 \mathrm{mg} / \mathrm{mL}$ and stored at $-80^{\circ} \mathrm{C}$ in a buffer containing $30 \mathrm{mM}$ HEPES, pH 7.5, $150 \mathrm{mM} \mathrm{NaCl}, 0.1 \mathrm{mM}$ EGTA, $1 \mathrm{mM}$ DTT.

\section{Crystallization and Structure Determination.}

FAK was incubated for 2 hours with $2 \mathrm{mM}$ adenosine $5^{\prime}$-triphosphate magnesium salt. Crystals were obtained using the hanging drop vapor diffusion method and equilibrating against $1 \mathrm{~mL}$ of the reservoir 
solution (0.2 M Magnesium formate dihydrate, $10-20 \%$ PEG 3350) at $20^{\circ} \mathrm{C}$. The protein was mixed 1:1 with the reservoir solution. For complex formation with the inhibitor, the crystals were transferred to a stabilizing solution (0.2 M Magnesium formate dihydrate, 30\% PEG 3350) containing $5 \mathrm{mM} 1$ and 5\% DMSO and were soaked for $24 \mathrm{~h}$. All data sets were collected at $100 \mathrm{~K}$ on beamline SLS X106 and processed with the XDS software package (Kabsch, 2010). The structures were solved by molecular replacement, using BUSTER (Bricogne et al., 2016). Model building was performed in Coot(Emsley, 2017), with compounds and waters fitted into the initial $\left|F_{o}\right|-\left|F_{c}\right|$ map, and the structures were refined using BUSTER. The coordinates of the holo-structures of FAK have been deposited in the RCSB Protein Data Bank. The refinement statistics and PDB accession codes are given in Table S2.

Protein -ligand interfaces were calculated using the PISA webserver (Krissinel and Henrick, 2007). Buried Surface Area (BSA, in $\AA^{2}$ ) and Accessible Surface Area (ASA, in $\AA^{2}$ ) were calculated per residue and normalized by dividing the BSA by ASA. Figures contain the interface profile of one chain of the asymmetric unit.

\section{Surface Plasmon Resonance.}

SPR measurements were performed on a Biacore 4000 instrument from GE Healthcare. Recombinant FAK [huFAK (410-689)] and PYK2 [huFAK (420-691)] were immobilized on a Biacore CM5 chip at $25^{\circ} \mathrm{C}$ at a flow rate of $10 \mu \mathrm{L} / \mathrm{min}$ using amine coupling at $\mathrm{pH} 4.50$ and $\mathrm{pH} 5.0$ respectively, according to Biacore's standard protocol. HBS-N (10 mM Hepes pH 7.40, $0.15 \mathrm{M} \mathrm{NaCl}, 0,05 \%$ Tween-20) served as running buffer during immobilization. FAK and PYK2 were applied at a concentration of $5 \mu \mathrm{g} / \mathrm{mL}$ in a buffer containing a 60 -fold excess of PF-431396. An unmodified carboxydextran matrix served as a reference surface. FAK and PYK2 inhibitors stored as $10 \mathrm{mM}$ stock solutions in 100\% dimethyl sulfoxide (DMSO) were dissolved in running buffer (20 mM HEPES pH 7.50, 150 mM NaCl, 0.05\% Tween 20, 1 mM DTT, 0.1 mM EDTA, 2\% DMSO) and analyzed using two-fold dilution series. Kinetic titration experiments were performed at $25^{\circ} \mathrm{C}$ with a flow rate of $30 \mu \mathrm{L} / \mathrm{min}$, a sample contact time of $120 \mathrm{~s}$ and a dissociation time between 300 and $600 \mathrm{~s}$. Data sets were processed and analyzed using the Biacore 4000 Evaluation software, version 1.1. Solvent corrected and double-referenced association and dissociation phase data were fitted to a simple 1:1 interaction model with mass transport limitations.

\section{NanoBRET cellular assays.}

The NanoBRET cellular target engagement assay is a tracer displacement assay. A BRET can here be observed between the NLuc (tagged to the full-length protein kinase) and the tracer molecule used (a commercial tracer that uses a BODIPY fluorophore) in living cells that were transiently transfected $20 \mathrm{~h}$ 
prior to assay performance. A compound of interest that binds to the protein kinase displaces the tracer and thereby reduces the BRET signal in a dose-dependent manner. The FAK and PYK2 NanoBRET assays that were performed here were conducted as described before (Röhm et al., 2019; Vasta et al., 2018). In brief: full-length FAK and PYK2 ORF (Promega) cloned in frame with a N-terminal NanoLuc-fusion and Cterminal NanoLuc-fusion, respectively, were transfected into HEK293T cells and proteins were allowed to express for $20 \mathrm{~h}$. For the target engagement assay serially diluted inhibitor and NanoBRET Kinase Tracer K5 (Promega) at $1 \mu \mathrm{M}$ for FAK and at $2 \mu \mathrm{M}$ for PYK2, respectively, were pipetted into white 384-well plates (Greiner 781 207). The corresponding FAK or PYK2-transfected cells were added and reseeded at a density of $2 \times 10^{5}$ cells $/ \mathrm{mL}$ after trypsinization and resuspending in Opti-MEM without phenol red (Life Technologies). The system was allowed to equilibrate for 2 hours at $37^{\circ} \mathrm{C} / 5 \% \mathrm{CO}_{2}$ prior to $\mathrm{BRET}$ measurements. To measure BRET, NanoBRET NanoGlo Substrate + Extracellular NanoLuc Inhibitor (Promega) was added as per the manufacturer's protocol, and filtered luminescence was measured on a PHERAstar plate reader (BMG Labtech) equipped with $450 \mathrm{~nm}$ BP filter (donor) and $610 \mathrm{~nm}$ LP filter (acceptor). Competitive displacement data were then graphed using GraphPad Prism software using a 4parameter curve fit with the following equation: Y=Bottom + (Top-Bottom) / $\left(1+10^{\wedge}((\right.$ LogIC50$\mathrm{X}) *$ HillSlope))

For the kinetic wash-out assay, the inhibitor at a concentration of 10 times its previously determined $/ C_{50}$ was pipetted into white 384-well plates (Greiner 781 207) and the corresponding FAK or PYK2-transfected cells were added and reseeded at a density of $2 \times 10^{5}$ cells $/ \mathrm{mL}$ after trypsinization and resuspending in Opti-MEM without phenol red (Life Technologies). The system was allowed to equilibrate for 2 hours at $37^{\circ} \mathrm{C} / 5 \% \mathrm{CO}_{2}$ to reach approximately $90 \%$ target occupancy prior to the wash-out via removal of the medium to extract any unbound inhibitor and addition of the removed volume of Opti-MEM medium. The tracer was added prior to BRET measurements at a final concentration as described for the target engagement assays. To measure BRET, NanoBRET NanoGlo Substrate + Extracellular NanoLuc Inhibitor (Promega) was added as per the manufacturer's protocol, and filtered luminescence was measured on a PHERAstar plate reader (BMG Labtech) equipped with $450 \mathrm{~nm}$ BP filter (donor) and $610 \mathrm{~nm}$ LP filter (acceptor) for $2 \mathrm{~h}$. The preincubated and saturated inhibitor-target complex dissociates during the washout and the tracer added associates which results in an increasing BRET signal. The Background of the kinetic data were corrected and then graphed using GraphPad Prism software using a one-phase association fit with the following equation: $\mathrm{Y}=\mathrm{YO}+($ Plateau- $\mathrm{YO}) *\left(1-\exp \left(-\mathrm{K}^{*} \mathrm{x}\right)\right)$ 


\section{NanoBRET plasmid mutation.}

Plasmids obtained by PROMEGA were point-mutated using Quickchange Mutagenesis with Hercll polymerase (Agilent). PCR cycles were prolonged to 35 cycles to obtain sufficient amounts of plasmid. PCR products were then transformed into E.coli MACH1 and potential clones sequenced for positive DNA sequence. DNA obtained was then amplified and purified using Midi-Prep Kits (QIAGEN) and used in the NanoBRET experiments as described above.

\section{Computations}

\section{Computational preparation of structures}

For FAK, we employed crystal structures for 8 complexes (compounds 1, 2, 3, 4, 5, 8, 10 and 12, PDB IDs 3bz3, 6yt6, 6yxv, 6yq1, 6yoj, 6yvs, 6yr9 and 6yvy, respectively). The structure of the complex with compound 11 was built by similarity with 1 (PDB 3bz3). The FAK-bound structures of compounds 7 and 9 were modelled using the complex with $\mathbf{8}$ as a template (PDB 6yvs). For compound 9, we employed both DFG loop-type and helix-type conformations of FAK to build complexes and performed simulations with both structures (see Fig. S4).

For PYK2, we employed crystal structures for 3 complexes (compounds 1, 10 and 12, PDB IDs 5TOB (Farand et al., 2016), 3FZR(Han et al., 2009) and 3H3C (Walker et al., 2008) , respectively). In the structures from PDB files 3FZR and 3H3C, there was a missing region (residues 573-584 and 571-584, respectively). This region was modeled using the Sphinx web server to model loops (Marks et al., 2017). The structure of PDB file 3FZR was used as input. The missing region in PDB file $3 \mathrm{H} 3 \mathrm{C}$ was modeled using PDB file $3 F Z R$ as a reference. In the structure from PDB file 5TOB, there were four regions missing (residues 446, 461-465, 495-496 and 570-587). Residues 570-587 were modeled using the RCD+ web server to model loops (LópezBlanco et al., 2016). The remaining regions were modeled using the PDB file 3FZR as a reference. Missing side chains were added using the WHATIF web server (Vriend, 1990). The protonation state of the histidine residues and charged residues at pH 7.4 was checked using pdb2pqr (Dolinsky et al., 2007; Dolinsky et al., 2004). To keep consistency among the different structures, their residues were modeled with the same protonation states. Residues were assigned unusual protonation states only when such a state appeared in two or more structures. GLU 617 was protonated because it had a pKa value higher than 7.4 in two different structures.

\section{Docking of compounds to PYK2}

Complexes for compounds without a crystal structure available were modeled using the protein coordinates and the ligand pose in the PDB file 3FZR as a reference. Compounds $\mathbf{4}$ and $\mathbf{5}$ had bulkier substitutions compared to $\mathbf{1 0}$ in 3FZR, so their positions were modeled by docking. Compound $\mathbf{2}$ had a 
substitution to a less bulky group, and its position was also modeled by docking. Docking of compounds $\mathbf{2}$, 4, and 5 to PYK2 (PDB 3FZR) was done with AutoDock Vina (Trott and Olson, 2010). Grids with $0.375 \AA$ spacing and 70 points along each side were centered in the known binding site. Rotation about bond torsions was allowed in ligands and the protein structure was kept frozen. The most favorable binding pose making two hydrogen bonds with Tyr 505 (known to be important interactions from crystal structures) was used for simulations.

\section{Modeling of PYK2 with a FAK-like DFG-motif helix}

A model of PYK2 with an FAK-like DFG-motif helix was built to understand why PYK2 is not able to form this structure. The structures of PYK2 (PDB 3FZR) and FAK (PDB 3BZ3) were aligned using PyMol and the coordinates of the atoms of the DFG-motif helix of FAK and residues nearby (residues 562-569) were used to replace the original coordinates in PYK2 (residues 565-572). Moreover, a mutation to Cys at position 462 was introduced in PYK2 to create the same disulfide bond present in FAK (residues 456 and 459 in FAK, residues 459 and 462 in PYK2). New rotamers for Glu 474 and Lys 457 in PYK2 were selected using PyMol to avoid steric clashes with the inserted DFG-motif helix. Missing residues 573-584 were modeled using the Sphinx web server to model loops (Marks et al., 2017). The structure of PYK2 containing the DFG-motif helix was used as input. Missing side chains were added using the WHATIF web server (Vriend, 1990). Crystallographic waters from PDB 3FZR were kept in the structure of PYK2 with the DFG-motif helix. The protonation states of the residues were kept the same as those defined for WT PYK2 (PDB 3FZR). To evaluate the effect on protein stability of the disulphide bridge that is located in the loop preceding the $\alpha$ helix C in FAK (C456-C459) but is not present in PYK2, we ran simulations twice, with and without the corresponding disulphide bridge. The simulations, however, showed only minor differences in the protein dynamics for the structures with and without disulphide bridges.

\section{MD and $\tau R A M D$ simulations}

The $\tau$ RAMD method for simulation of dissociation trajectories of protein ligand complexes and computation of relative residence times has been reported elsewhere (Kokh et al., 2018), where the method was assessed on $70 \mathrm{~N}-\mathrm{HSP} 90$ ATP-pocket binding compounds, and we used the same method and parameterization. Here, we will give a brief description of the procedure (a complete workflow is illustrated in Fig. S7 (Panel A was prepared using weblogo web server (Crooks et al., 2004)).

We employed the Gaff force field (Wang et al., 2004) for ligands and the Amber ff14 force field (Maier et al., 2015) for the protein. RESP (Cornell et al., 2002) partial atomic charges for ligands were obtained using molecular electrostatic potentials from ab initio quantum mechanical calculations performed at the $\mathrm{HF}$ level with a 6-31G*(1d) basis set using the GAMESS software (Gordon and Schmidt, 2005). 
First, the system was solvated in a periodic box of TIP3P water molecules (Jorgensen, 1983) with $\mathrm{NaCl}$ ion concentration of $150 \mathrm{mM}$. Then it was energy minimized, gradually heated and equilibrated stepwise with decreasing harmonic restraints on all heavy atoms of the protein and ligand using the AMBER15 software (Case et al., 2015). Then the system was run for 10 ns without restraints and the final snapshot was used as an input for equilibration simulations carried out with the NAMD (Phillips et al., 2005) simulation software. The equilibration was performed in the NPT ensemble using the Langevin and Nosé-Hoover methods for the temperature (300K) and pressure (1 atm) control, respectively. At least four replicas of equilibration simulations (20ns each) were prepared for each ligand.

The last snapshot of each replica was used to simulate ligand dissociation. For this, the RAMD method (Lüdemann et al., 2000) was applied, where an additional force with a magnitude of $13 \mathrm{kcal} / \mathrm{mol} \AA$ and a random direction was applied to the center of mass of the ligand in MD simulations. Every $100 \mathrm{fs}$, the force direction was changed randomly if the ligand center of mass did not move further than a threshold of $0.025 \AA$ and was retained otherwise. Simulations were stopped if the ligand center of mass moved further than $30 \AA$ from the original position. At least 10 RAMD dissociation trajectories were generated starting with each equilibration replica. The times required for ligand dissociation in each trajectory were stored and used for computation of the relative residence times as described in (Kokh et al., 2018) and illustrated in Fig. S7.

Additionally, we repeated computations with a random force magnitude of $8 \mathrm{kcal} / \mathrm{mol} \AA$ for the fast dissociating compounds only ( $k_{\text {off }}>1 . e-3 \mathrm{~s}^{-1}$, compounds $2,6,7,8,9$ and 12 with FAK and all compounds with PYK2) in order to sample the egress process more slowly. However, we did not obtain a significant improvement in the correlation with SPR data.

Conventional MD simulations for the apo-protein structures were performed using the same procedure and parameters as described above for the equilibration step of the $\tau$ RAMD workflow. For each protein, three 500 ns trajectories were simulated.

\section{Analysis of MD trajectories}

To analyse the protein-ligand interactions in the complexes, we computed protein-ligand interaction fingerprints, IFP, for the last 500 frames of each compound and equilibration trajectory using the MD-IFP tools (Kokh et al., 2020). In this approach, the IFP include hydrophobic interactions, salt-links, hydrogen bonds, halogen bonds, aromatic interactions and water-mediated protein-ligand bridges, as well as all unspecific contacts within a threshold of $5 \AA$ (aromatic interactions and salt bridges make very minor contributions and, therefore, are not presented in plots). From these data, the occurrence of each type of 
interaction was obtained for each compound and the binding site protein residues. For the computation of BSA, the occurrence of unspecific protein-ligand contacts was employed.

\section{Quantification and statistical analysis}

\section{Surface-Plasmon-Resonance Data}

Data from at least $\mathrm{n}=5$ individual experiments are reported as $k_{\mathrm{on}}, k_{\mathrm{off}}, K_{\mathrm{D}}$ average \pm SD. For analysis, the Biacore 4000 Evaluation software, version 1.1 was used for solvent correction and double-referenced association and dissociation phase data fitting to a simple 1:1 interaction model with mass transport limitations.

\section{NanoBRET Data}

Data from at least $\mathrm{n}=3$ individual experiments are reported as $K_{1}, k_{\text {obs }}$ average $\pm \mathrm{SEM}$. Analysis for $I C_{50}$ determination was performed using GraphPad Prism 8 software using a 4-parameter curve fit with the following equation: $\mathrm{Y}=$ Bottom $+($ Top-Bottom $) /\left(1+10^{\wedge}((\right.$ LogIC50-X)*HillSlope $))$. $I C_{50}$ S were then converted to $K_{\mathrm{l} \text {, obs }}$ using the Cheng-Prusoff-Relation(Cheng and Prusoff, 1973) taking the tracer parameters and tracer concentration into account. Analysis for $k_{\text {obs }}$ determination was performed using GraphPad Prism 8 software using a one-phase association fit with the following equation: $Y=Y 0+($ Plateau-Y0)*(1-exp(-K* $\mathrm{x})$ ).

\section{References}

Allen, J.G., Lee, M.R., Han, C.-Y.E., Scherrer, J., Flynn, S., Boucher, C., Zhao, H., O'Connor, A.B., Roveto, P., Bauer, D., et al. (2009). Identification of small molecule inhibitors of proline-rich tyrosine kinase 2 (Pyk2) with osteogenic activity in osteoblast cells. Bioorganic \& medicinal chemistry letters 19, 4924-4928.

Arcari, J.T., Bhattacharya, S.K., Brosius, A.D., Luzzio, M.J., Nelson, K.L., Pan, G., Southers Jr., J.A., Wishka, D.G., and Xiao, J. (2006). Pyrimidine derivatives for the treatment of abnormal cell growth (WO2007072158A2).

Avraham, H., Park, S.-Y., Schinkmann, K., and Avraham, S. (2000). RAFTK/Pyk2-mediated cellular signalling. Cellular Signalling 12, 123-133.

Bricogne, G., Blanc, E., Brandl, M., Flensburg, C., Keller, P., Paciorek, W., Roversi, P., Sharff, A., Smart, O., and Vonrhein, C. (2016). BUSTER (Global Phasing Ltd., Cambridge, UK). Version XYZ.

Buckbinder, L., Crawford, D.T., Qi, H., Ke, H.Z., Olson, L.M., Long, K.R., Bonnette, P.C., Baumann, A.P., Hambor, J.E., and Grasser, W.A. (2007). Proline-rich tyrosine kinase 2 regulates osteoprogenitor cells and bone formation, and offers an anabolic treatment approach for osteoporosis. Proceedings of the National Academy of Sciences 104, 10619-10624.

Case, D., Berryman, J., Betz, R., Cerutti, D., Cheatham lii, T., Darden, T., Duke, R., Giese, T., Gohlke, H., and Goetz, A. (2015). AMBER 2015.

Cheng, Y.-C., and Prusoff, W.H. (1973). Relationship between the inhibition constant (K1) and the concentration of inhibitor which causes 50 per cent inhibition (150) of an enzymatic reaction. Biochemical pharmacology 22, 3099-3108.

Copeland, R.A. (2016). The drug-target residence time model: A 10-year retrospective. Nature Reviews Drug Discovery 15, 87. 
Cornell, W.D., Cieplak, P., Bayly, C.I., and Kollman, P.A. (2002). Application of RESP charges to calculate conformational energies, hydrogen bond energies, and free energies of solvation. Journal of the American Chemical Society 115, 9620-9631.

Crooks, G.E., Hon, G., Chandonia, J.-M., and Brenner, S.E. (2004). WebLogo: a sequence logo generator. Genome research 14, 1188-1190.

Dolinsky, T.J., Czodrowski, P., Li, H., Nielsen, J.E., Jensen, J.H., Klebe, G., and Baker, N.A. (2007). PDB2PQR: expanding and upgrading automated preparation of biomolecular structures for molecular simulations. Nucleic acids research 35, W522-W525.

Dolinsky, T.J., Nielsen, J.E., McCammon, J.A., and Baker, N.A. (2004). PDB2PQR: an automated pipeline for the setup of Poisson-Boltzmann electrostatics calculations. Nucleic acids research 32, W665-W667.

Emsley, P. (2017). Tools for ligand validation in Coot. Acta Crystallographica Section D: Structural Biology 73, 203-210.

Fan, H., and Guan, J.-L. (2011). Compensatory function of Pyk2 protein in the promotion of focal adhesion kinase (FAK)-null mammary cancer stem cell tumorigenicity and metastatic activity. The Journal of biological chemistry 286, 18573-18582.

Farand, J., Mai, N., Chandrasekhar, J., Newby, Z.E., Van Veldhuizen, J., Loyer-Drew, J., Venkataramani, C., Guerrero, J., Kwok, A., Li, N., et al. (2016). Selectivity switch between FAK and Pyk2: Macrocyclization of FAK inhibitors improves Pyk2 potency. Bioorg Med Chem Lett 26, 5926-5930.

Gordon, M.S., and Schmidt, M.W. (2005). Advances in electronic structure theory: GAMESS a decade later. In Theory and applications of computational chemistry (Elsevier), pp. 1167-1189.

Han, S., Mistry, A., Chang, J.S., Cunningham, D., Griffor, M., Bonnette, P.C., Wang, H., Chrunyk, B.A., Aspnes, G.E., Walker, D.P., et al. (2009). Structural characterization of proline-rich tyrosine kinase 2 (PYK2) reveals a unique (DFG-out) conformation and enables inhibitor design. J Biol Chem 284, 13193-13201.

Heinrich, T., Seenisamy, J., Emmanuvel, L., Kulkarni, S.S., Bomke, J., Rohdich, F., Greiner, H., Esdar, C., Krier, M., Gradler, U., et al. (2013). Fragment-based discovery of new highly substituted $1 \mathrm{H}$-pyrrolo[2,3-b]- and $3 \mathrm{H}$-imidazolo[4,5-b]-pyridines as focal adhesion kinase inhibitors. J Med Chem 56, 1160-1170.

Jones, S., Shapiro, G., Bendell, J., Chen, E., Bedard, P., Cleary, J., Pandya, S., Pierce, K., Houk, B., and Hosea, N. (2011). Phase I study of PF-04554878, a second-generation focal adhesion kinase (FAK) inhibitor, in patients with advanced solid tumors. Journal of clinical oncology 29, 3002-3002.

Jorgensen, W. (1983). I. Am. Chem. Sot. 106 (1984) 6638. WL Jorgensen, J. Chandrasekhar, JD Madura, RW Impey and ML Klein. J Chem Phys 79, 0.

Kabsch, W. (2010). Xds. Acta Crystallographica Section D: Biological Crystallography 66, 125-132.

Kath, J.C., and Luzzio, M.J. (2003). Pyrimidine derivates for the treatment of abnormal cell growth (WO2004056786A2).

Kokh, D.B., Amaral, M., Bomke, J., Grädler, U., Musil, D., Buchstaller, H.-P., Dreyer, M.K., Frech, M., Lowinski, M., Vallee, F., et al. (2018). Estimation of Drug-Target Residence Times by $\tau$-Random Acceleration Molecular Dynamics Simulations. Journal of chemical theory and computation 14, 3859-3869.

Kokh, D.B., Doser, B., Richter, S., Ormersbach, F., Cheng, X., and Wade, R.C. (2020). A Workflow for Exploring Ligand Dissociation from a Macromolecule: Efficient Random Acceleration Molecular Dynamics Simulation and Interaction Fingerprints Analysis of Ligand Trajectories. J Chem Phys 153.

Krissinel, E., and Henrick, K. (2007). Inference of macromolecular assemblies from crystalline state. Journal of molecular biology 372, 774-797.

Lietha, D., and Eck, M.J. (2008). Crystal structures of the FAK kinase in complex with TAE226 and related bis-anilino pyrimidine inhibitors reveal a helical DFG conformation. PLoS One 3, e3800.

Lipinski, C.A., and Loftus, J.C. (2010). Targeting Pyk2 for therapeutic intervention. Expert opinion on therapeutic targets 14, 95-108.

López-Blanco, J.R., Canosa-Valls, A.J., Li, Y., and Chacón, P. (2016). RCD+: Fast loop modeling server. Nucleic acids research 44, W395-W400. 
Lüdemann, S.K., Lounnas, V., and Wade, R.C. (2000). How do substrates enter and products exit the buried active site of cytochrome P450cam? 2. Steered molecular dynamics and adiabatic mapping of substrate pathways. Journal of molecular biology 303, 813-830.

Luzzio, M.J., Autry, C.L., Bhattacharya, S.K., Freeman-Cook, K.D., Hayward, M.M., Hulford, C.A., Nelson, K.L., Xiao, J., and Zhao, X. (2008). Sulfonyl amide derivatives for the treatment of abnormal cell growth (WO2008129380A1).

LV, P.C., Jiang, A.Q., Zhang, W.M., and Zhu, H.L. (2018). FAK inhibitors in Cancer, a patent review. Expert Opin Ther Pat 28, 139-145.

Maier, J.A., Martinez, C., Kasavajhala, K., Wickstrom, L., Hauser, K.E., and Simmerling, C. (2015). ff14SB: improving the accuracy of protein side chain and backbone parameters from ff99SB. Journal of chemical theory and computation 11, 3696-3713.

Marks, C., Nowak, J., Klostermann, S., Georges, G., Dunbar, J., Shi, J., Kelm, S., and Deane, C.M. (2017). Sphinx: merging knowledge-based and ab initio approaches to improve protein loop prediction. Bioinformatics 33, 1346-1353.

Matsuhisa, A., Kinoyama, I., Toyoshima, A., Nakahara, T., Takeuchi, M., and Okada, M. (2001). Fused imidazolium derivatives (US20030114508A1).

Phillips, J.C., Braun, R., Wang, W., Gumbart, J., Tajkhorshid, E., Villa, E., Chipot, C., Skeel, R.D., Kale, L., and Schulten, K. (2005). Scalable molecular dynamics with NAMD. Journal of computational chemistry 26, 1781-1802.

Robers, M.B., Dart, M.L., Woodroofe, C.C., Zimprich, C.A., Kirkland, T.A., Machleidt, T., Kupcho, K.R., Levin, S., Hartnett, J.R., Zimmerman, K., et al. (2015). Target engagement and drug residence time can be observed in living cells with BRET. Nature communications 6, 10091.

Roberts, W.G., Ung, E., Whalen, P., Cooper, B., Hulford, C., Autry, C., Richter, D., Emerson, E., Lin, J., Kath, J., et al. (2008). Antitumor activity and pharmacology of a selective focal adhesion kinase inhibitor, PF562,271. Cancer Res 68, 1935-1944.

Röhm, S., Berger, B.-T., Schröder, M., Chaikuad, A., Winkel, R., Hekking, K.F., Benningshof, J.J., Müller, G., Tesch, R., and Kudolo, M. (2019). Fast Iterative Synthetic Approach toward Identification of Novel Highly Selective p38 MAP Kinase Inhibitors. Journal of medicinal chemistry 62, 10757-10782.

Schlaepfer, D.D., Hauck, C.R., and Sieg, D.J. (1999). Signaling through focal adhesion kinase. Progress in biophysics and molecular biology 71, 435-478.

Schuetz, D.A., Witte, W.E.A.d., Wong, Y.C., Knasmueller, B., Richter, L., Kokh, D.B., Sadiq, S.K., Bosma, R., Nederpelt, I., and Heitman, L.H. (2017). Kinetics for Drug Discovery: An industry-driven effort to target drug residence time. Drug Discovery Today 22, 896-911.

Slack-Davis, J.K., Martin, K.H., Tilghman, R.W., Iwanicki, M., Ung, E.J., Autry, C., Luzzio, M.J., Cooper, B., Kath, J.C., Roberts, W.G., et al. (2007). Cellular characterization of a novel focal adhesion kinase inhibitor. J Biol Chem 282, 14845-14852.

Tai, Y.-L., Chen, L.-C., and Shen, T.-L. (2015). Emerging roles of focal adhesion kinase in cancer. BioMed research international 2015, 690690 .

Trott, O., and Olson, A.J. (2010). AutoDock Vina: improving the speed and accuracy of docking with a new scoring function, efficient optimization, and multithreading. Journal of computational chemistry $31,455-$ 461.

Tse, K.W.K., Lin, K.B.L., Dang-Lawson, M., Guzman-Perez, A., Aspnes, G.E., Buckbinder, L., and Gold, M.R. (2012). Small molecule inhibitors of the Pyk2 and FAK kinases modulate chemoattractant-induced migration, adhesion and Akt activation in follicular and marginal zone B cells. Cellular immunology 275, 47-54.

Vasta, J.D., Corona, C.R., Wilkinson, J., Zimprich, C.A., Hartnett, J.R., Ingold, M.R., Zimmerman, K., Machleidt, T., Kirkland, T.A., Huwiler, K.G., et al. (2018). Quantitative, Wide-Spectrum Kinase Profiling in Live Cells for Assessing the Effect of Cellular ATP on Target Engagement. Cell Chem Biol 25, 206-214 e211. 
Vriend, G. (1990). WHAT IF: a molecular modeling and drug design program. Journal of molecular graphics 8, 52-56.

Walker, D.P., Bi, F.C., Kalgutkar, A.S., Bauman, J.N., Zhao, S.X., Soglia, J.R., Aspnes, G.E., Kung, D.W., KlugMcLeod, J., Zawistoski, M.P., et al. (2008). Trifluoromethylpyrimidine-based inhibitors of proline-rich tyrosine kinase 2 (PYK2): structure-activity relationships and strategies for the elimination of reactive metabolite formation. Bioorg Med Chem Lett 18, 6071-6077.

Walker, D.P., Zawistoski, M.P., McGlynn, M.A., Li, J.-C., Kung, D.W., Bonnette, P.C., Baumann, A., Buckbinder, L., Houser, J.A., and Boer, J. (2009). Sulfoximine-substituted trifluoromethylpyrimidine analogs as inhibitors of proline-rich tyrosine kinase 2 (PYK2) show reduced hERG activity. Bioorganic \& medicinal chemistry letters 19, 3253-3258.

Wang, J., Wolf, R.M., Caldwell, J.W., Kollman, P.A., and Case, D.A. (2004). Development and testing of a general amber force field. Journal of computational chemistry 25, 1157-1174.

Wendt, M.K., Schiemann, B.J., Parvani, J.G., Lee, Y.-H., Kang, Y., and Schiemann, W.P. (2013). TGF- $\beta$ stimulates Pyk2 expression as part of an epithelial-mesenchymal transition program required for metastatic outgrowth of breast cancer. Oncogene 32, 2005-2015. 